\title{
A computational paradigm for dynamic logic-gates in neuronal activity
}

\author{
Amir Goldental ${ }^{1 \dagger}$, Shoshana Guberman ${ }^{1,2+},{\text { Roni } \text { Vardi }^{2 \dagger} \text { and Ido Kanter }}^{1,2 *}$ \\ 1 Department of Physics, Bar-llan University, Ramat-Gan, Israel \\ ${ }^{2}$ The Goodman Faculty of Life Sciences, Gonda Interdisciplinary Brain Research Center, Bar-Ilan University, Ramat-Gan, Israel
}

\section{Edited by:}

Gabriel A. Silva, University of

California, San Diego, USA

Reviewed by:

Paolo Del Giudice, Italian National Institute of Health, Italy

Yasuhiro Tsubo, Ritsumeikan

University, Japan

*Correspondence:

Ido Kanter, Gonda Interdisciplinary Brain Research Center, Bar-llan

University, Ramat-Gan 52900, Israel e-mail: ido.kanter@gmail.com

${ }^{\dagger}$ These authors have contributed equally to this work.
In 1943 McCulloch and Pitts suggested that the brain is composed of reliable logic-gates similar to the logic at the core of today's computers. This framework had a limited impact on neuroscience, since neurons exhibit far richer dynamics. Here we propose a new experimentally corroborated paradigm in which the truth tables of the brain's logic-gates are time dependent, i.e., dynamic logic-gates (DLGs). The truth tables of the DLGs depend on the history of their activity and the stimulation frequencies of their input neurons. Our experimental results are based on a procedure where conditioned stimulations were enforced on circuits of neurons embedded within a large-scale network of cortical cells in-vitro. We demonstrate that the underlying biological mechanism is the unavoidable increase of neuronal response latencies to ongoing stimulations, which imposes a non-uniform gradual stretching of network delays. The limited experimental results are confirmed and extended by simulations and theoretical arguments based on identical neurons with a fixed increase of the neuronal response latency per evoked spike. We anticipate our results to lead to better understanding of the suitability of this computational paradigm to account for the brain's functionalities and will require the development of new systematic mathematical methods beyond the methods developed for traditional Boolean algebra.

Keywords: neuronal circuit, logic-gates, neuronal response latency, in vitro modular networks, Boolean algebra

\section{INTRODUCTION}

This year we are celebrating the 70th anniversary of the publication of the seminal work by Warren S. McCulloch, a neuroscientist, and Walter Pitts, a logician, entitled "A logical calculus of the ideas immanent in nervous activity" (Mcculloch and Pitts, 1943). They attempted to understand how the brain could produce highly complex patterns by using many interconnected building blocks of the brain, the neurons. In their model, the brain is composed of Boolean entities functioning as threshold units. Such simplified units constitute pure and reliable logicgates (e.g., AND, XOR), similar to the logic at the core of today's computers. The generalization of this simplified Boolean framework to include unreliable elements has emerged in 1956 by the innovative work of John von Neumann (Von Neumann, 1956). These concepts as well as the earlier pioneering work of Claude Shannon to simplify Boolean circuits (Shannon, 1938) are at the cornerstone of today's computational paradigm (Turing, 1938).

The computational framework of McCulloch and Pitts had a tremendous impact on the development of artificial neural networks (Hopfield, 1982; Krogh, 2008; Qian et al., 2011; Gerstner et al., 2012; Gilja et al., 2012) and machine learning theory (Sutton and Barto, 1998; Hunt et al., 2012). Their concept triggered the next major development in theoretical neural networks when in 1958 Frank Rosenblatt introduced the concept of the perceptron (Rosenblatt, 1958), the prototypical linear classifier, which ever since has been theoretically investigated and generalized to more structured multi-layer and recurrent architectures
(Litwin-Kumar and Doiron, 2012; Stoianov and Zorzi, 2012). Nevertheless, it is fair to conclude that the concept of simplified Boolean neurons had a limited impact on neuroscience, which exhibit much richer temporal dynamics (Izhikevich, 2006; Izhikevich and Hoppensteadt, 2009; Gal et al., 2010; Vardi et al., 2012a). Moreover, it appears that the brain is the most ineffective environment to implement such a Boolean logical operating system, comprised of static logic-gates (SLGs).

Seven decades after the proposed neuronal paradigm by McCulloch and Pitts, the fundamental concept of the computational abilities of the nervous system remains unclear (Hodges, 2012). On the one hand, one might conclude that the search for a comprehensive computational logic framework is irrelevant, as specialization in specific behavioral and perceptual tasks requires different "operating systems." On the other hand, it is evident that the "hardware" implementations of all complex brain tasks are composed of similar basic interconnected building blocks (neurons) having many features in common, which are enhanced and possibly dominant when operating as an ensemble (Abeles, 1991).

In the present study, we extend the recently demonstrated new experimentally corroborated paradigm in which the logical operations of the brain differ from the logic of computers (Vardi et al., 2013b). Unlike a burned logic-gate on a designed chip that consistently follows the same truth-table, here the functionality of the brain's logic-gates depend on the history of their activity, the stimulation frequencies of their input neurons, as well as on the activity of their interconnections. Our results are based 
on an experimental procedure where conditioned stimulations were enforced on circuits of neurons embedded within a largescale network of cortical cells in-vitro (Marom and Shahaf, 2002; Morin et al., 2005; Wagenaar et al., 2006; Vardi et al., 2012b). We demonstrate that the underlying biological mechanism is the unavoidable increase of neuronal response latencies to ongoing stimulations (Aston-Jones et al., 1980; De Col et al., 2008; Ballo and Bucher, 2009; Gal et al., 2010; Soudry and Meir, 2012), which imposes a non-uniform gradual stretching of delays associated with the neuronal circuit (Kanter et al., 2011; Vardi et al., 2012a, 2013a,c). To further support and expand the limited experimental results, we present a straightforward theoretical model based on the assumption of identical neurons with a constant increase in their neuronal response latency per evoked spike. This model, corroborated with simulations, allows us to explore the behavior of more complex structured neuronal DLGs in addition to SLG (Vogels and Abbott, 2005). We anticipate our results to be a starting point for larger scale in-vitro experiments and structured recurrent neuronal circuits, which will lead to a better understanding of the suitability of this computational paradigm to
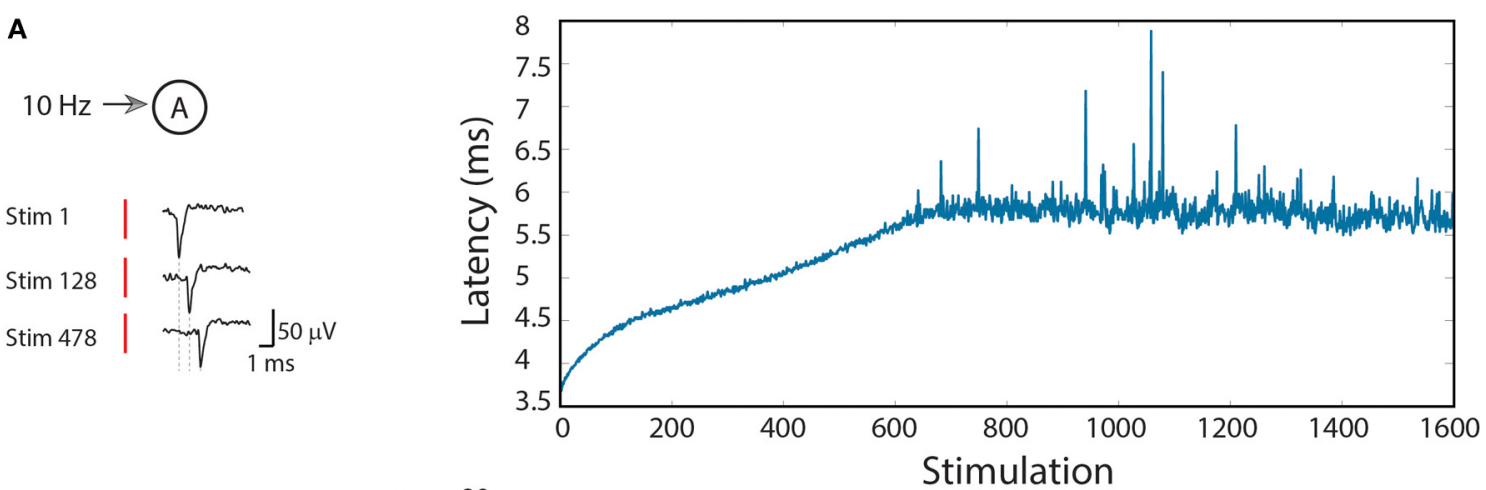

B

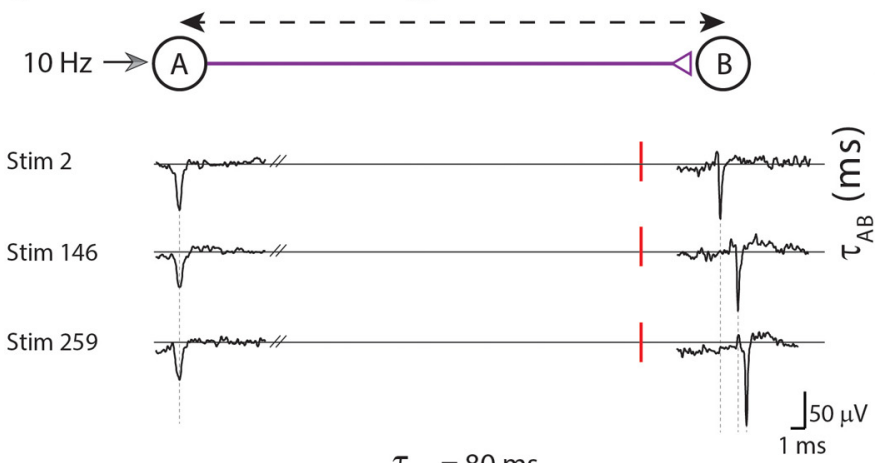
$\tau_{\mathrm{AE}}=80 \mathrm{~ms}$

C<smiles>OCc1cc2c3cc(ccc4ccccc14)C(=CC=2)C=Cc1ccccc1C=3</smiles>

Stim 1

Stim 56

Stim 265

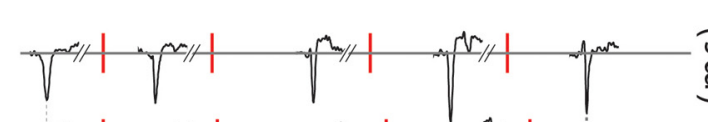

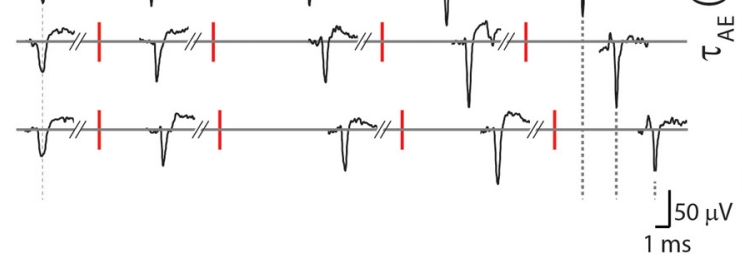

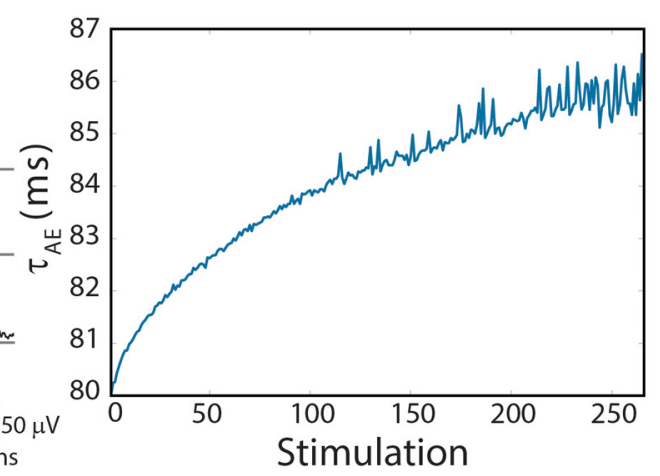

FIGURE 1 | (Color online) Stretching of the neuronal response latency to ongoing stimulations. (A) An extracellular stimulation of a single neuron at a rate of $10 \mathrm{~Hz}$. The relative time-gap between a stimulation (red bar) and its corresponding recorded evoked spike (voltage minima), the neuronal response latency, is exemplified for several stimulations (left). The graph (right) summarizes the response latencies over 1600 stimulations. (B) A two-neuron-chain where neuron $A$ is stimulated at a rate of $10 \mathrm{~Hz}$, and the initial delay between evoked spikes of neurons $A$ and $B$ is set to $\tau_{A B}=80 \mathrm{~ms}$. Several recorded spikes from neurons $A$ and $B$ are exemplified (left). The graph (right) summarizes the $\sim 2 \mathrm{~ms}$ increase in $\tau_{\mathrm{AB}}$ over $\sim 270$ stimulations. (C) Similar to (B) but with a five-neuron-chain, and a $\sim 6 \mathrm{~ms}$ increase in $\tau_{\mathrm{AE}}$ which accumulates the stretching of all four (B-E) neuronal response latencies. Reproduced upon permission from Vardi et al. (2013b). 
account for the brain's functionalities. In addition, this paradigm will require the development of new systematic methods and practical tools beyond the methods developed for traditional Boolean algebra (Chavesa et al., 2005; Nahin, 2012).

\section{ELASTIC RESPONSE LATENCY SINGLE NEURON}

The neuronal response latency, measured as the time-lag between a stimulation and its corresponding evoked spike, is one of the most significant time-dependent features at the single neuron level, and typically it is on the order of several milliseconds (Eccles et al., 1966; Van Pelt et al., 2004; Ballo and Bucher, 2009; Gal et al., 2010; Vardi et al., 2012a). When stimulated repeatedly, a neuron exhibits a tendency to gradually stretch its stimulus-response delay over few milliseconds (Spira et al., 1976; Grossman et al., 1979; Thomson and West, 1993; Tal et al., 2001; Fuhrmann et al., 2002; Bakkum et al., 2008; Scroggs, 2008).

To exemplify this neuronal feature, stimulations at a rate of $10 \mathrm{~Hz}$ (Figure 1A; Vardi et al., 2013b) were given to cultured cortical neurons that were functionally isolated from their network by pharmacological blockers of both excitatory and inhibitory synapses (see Supplementary Material). The stimulated neuron responded with a very high reliability, resulting in a typical increase of a few milliseconds in the response latency over a few hundreds of repeated stimulations (Figure 1A; Vardi et al., 2013b). Results indicate that the neuronal response latency increases by a few $\mu$ s per evoked spike, which represents a finer time scale of cortical dynamics, $\mu \mathrm{s}$, as discussed at (Vardi et al., 2012a). Specifically, one might notice three main trends of the response latency increase. For the first several stimulations there is a large increase in the neuronal response latency, in the order of several dozen $\mu$ s per evoked spike (Figure 1A; Vardi et al., 2013b).
This state is followed by a fast decay to the second state, where the average increase in the neuronal response latency per evoked spike is only several $\mu \mathrm{s}$, and the stretching of the neuronal response latency is roughly linear. The second state is the main contributor to the latency increase and lasts for a relatively long section of the stimulation period. In the presented experiment the second state starts after $\sim 100$ stimulations and lasts for approximately 550 stimulations, periods which vary across different neurons. Finally the neuron enters the third state, known as the intermittency phase (Gal et al., 2010; Vardi et al., 2012a), characterized by fluctuations around an average latency (starts after $\sim 650$ stimulation in the presented experiment). An apparent increase in the neuronal response latency to periodic stimulations can be observed for stimulation rates higher than $\sim 3 \mathrm{~Hz}$. Typically, the higher the stimulation rate, the larger the average increase of the response latency per evoked spike (Gal et al., 2010; Vardi et al., 2012a). This process is a fully reversible phenomenon and after a waiting time of a few seconds without stimulations, the response latency substantially decays and in a timescale of several minutes the initial response latency is completely restored.

The approximately linear increase in the neuronal response latency per evoked spike before entering the intermittent stage is at the center of our study. Consequently, the proposed theoretical methods are based on the approximation that the neuronal response latency increases by a constant value $(\Delta)$ per evoked spike (identical for all neurons and time-independent).

\section{CIRCUIT LEVEL}

To analyze the impact of dynamic neuronal response latency at a circuit level, we artificially generated conditioned stimulations over a circuit of neurons embedded within a large scale network of cortical cells in-vitro (see Supplementary Material). Our

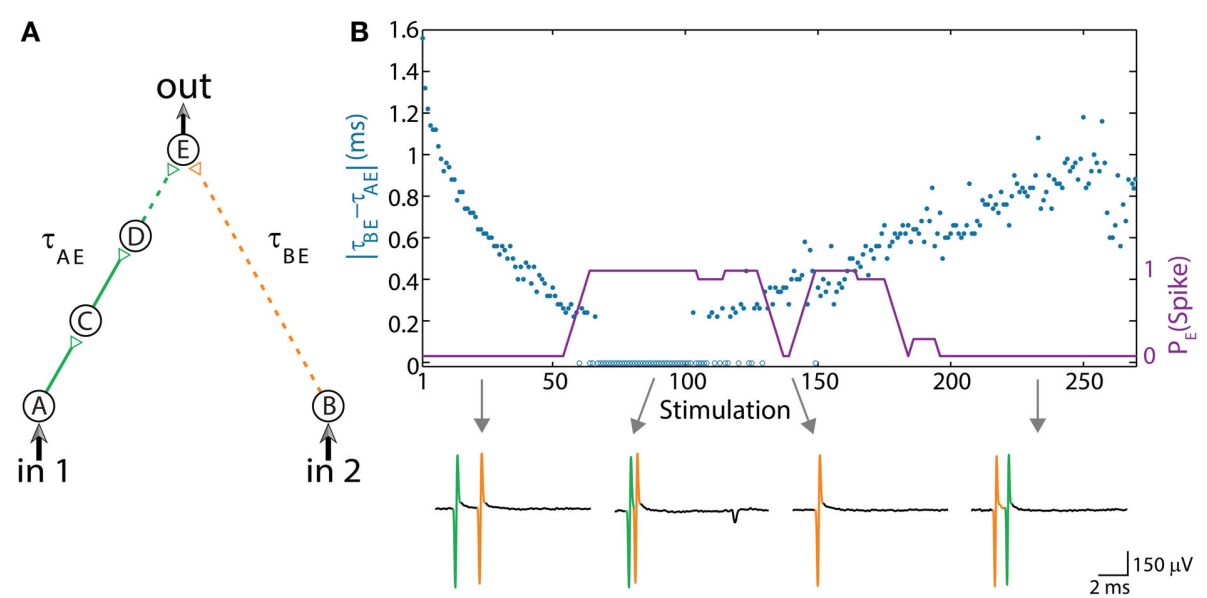

FIGURE 2 | (Color online) Dynamic AND gate. (A) Schematic of an AND-gate consisting of five neurons and weak/strong stimulations (sub/above threshold) represented by dashed/full lines. (B) The delays are initially set to $\tau_{\mathrm{BE}}=80 \mathrm{~ms}$ and $\tau_{\mathrm{AE}} \approx \tau_{\mathrm{BE}}-1.6 \mathrm{~ms}$ (in the presented experiment the initial delays between consecutive neurons in the left chain were selected to be equal, however, results are robust to arbitrary delays summing up to $\tau_{\mathrm{AE}}$ ). Applying simultaneous stimulations at $\sim 10 \mathrm{~Hz}$ to the input neurons, the two delays become the same and later reverse roles where $\tau_{\mathrm{AE}} \approx \tau_{\mathrm{BE}}+1 \mathrm{~ms}$, as presented by the blue circles as a function of the stimulation number. Unified longer stimulations were given for events where $\left|\tau_{\mathrm{AE}}-\tau_{\mathrm{BE}}\right|<200 \mu \mathrm{s}$ and are presented by zero time-lag open blue circles (Methods in Supplementary Material). The probability of an evoked spike of neuron $\mathrm{E}$ over a sliding window of 10 stimulations is presented by the purple line. Different segments of the voltage recordings of neuron $E$ are exemplified below, the arrows point from different scenarios to their matching recordings. Reproduced upon permission from Vardi et al. (2013b). 
first experimental design consisted of a chain of two neurons (Figure 1B; Vardi et al., 2013b). Neuron A is stimulated at a rate of $10 \mathrm{~Hz}$ and the initial time-gap between consecutive evoked spikes of neurons $\mathrm{A}$ and $\mathrm{B}$ is set to $\tau_{\mathrm{AB}}=80 \mathrm{~ms}$ [neuron $\mathrm{B}$ is stimulated $80-\mathrm{L}_{B}(0)$ ms after an evoked spike of neuron $A$, where $L_{B}(0)$ stands for the initial response latency of neuron B] (Figure 1B; Vardi et al., 2013b). After $\sim 270$ stimulations the response latency of neuron $B$ increases by $\sim 2 \mathrm{~ms}$, thus resulting in an increase of the delay, $\tau_{\mathrm{AB}} \approx 82 \mathrm{~ms}$.

The increase in the delays of the neuronal chain has an accumulative effect, as a result of the increase in the neuronal response latencies of the neurons comprising the chain (Figure 1C; Vardi et al., 2013b). More neurons in a chain lead to a faster and greater increase of the entire delay of the chain. In order to compare results of two-neuron and five-neuron chains, a chain of five neurons $(\mathrm{A}, \mathrm{B}, \mathrm{C}, \mathrm{D}, \mathrm{E})$ was examined. $\tau_{\mathrm{AE}}$ was set to $80 \mathrm{~ms}$, resulting in an initial time-gap of $80 \mathrm{~ms}$ between evoked spikes of neurons $\mathrm{A}$ and $\mathrm{E}$, where $\tau_{\mathrm{AE}}=\tau_{\mathrm{AB}}+\tau_{\mathrm{BC}}+\tau_{\mathrm{CD}}+\tau_{\mathrm{DE}}$. In the presented experiment the initial delays between consecutive neurons were selected to be equal, however, results are robust to arbitrary delays summing up to $\tau_{\mathrm{AE}}$. After $\sim 270$ stimulations of neuron $\mathrm{A}$, where each stimulation results in an evoked spike of neuron $\mathrm{E}$, the stretching of $\tau_{\mathrm{AE}}$ is about $6 \mathrm{~ms}$ (Figure 1C; Vardi et al., 2013b).

It is evident that the total delay stretching of a five-neuron chain is superior to that of a two-neuron chain, as the stretching of each individual neuron is accumulative. The experimentally corroborated paradigm presented below is based on this key feature of the unavoidable accumulated stretching, enabling the implementation of different types of DLGs in the brain.

\section{EXPERIMENTALLY EXAMINED DLGs}

Neuronal logic-gates consist of a multilayer feedforward neural network, with a single output neuron. In this study we differentiate between two main classes of logic-gates, SLGs and DLGs. For illustration, a typical static neuronal AND-gate would consist of two input neurons and an output neuron which fires if and only if both input neurons are stimulated simultaneously. However, a dynamic AND-gate would change its functionality over time.

\section{DYNAMIC AND-GATE}

The first experimentally examined feedforwad neuronal circuit is a dynamic AND-gate consisting of five neurons and 6 conditional stimulations, which split to weak/strong stimulations represented by dashed/full lines (Figure 2A; Vardi et al., 2013b). A strong stimulation (above threshold) is characterized by a high amplitude and/or long duration, resulting in a reliable response. In contrary, a weak stimulation (sub threshold) is characterized by a lower amplitude and/or shorter duration, resulting in an evoked spike only in case of spatial or temporal summation, where the time-lag between two consecutive weak stimulations is short enough, as discussed in Vardi et al. (2013b).

The delay of the three-neuron chain, $\tau_{\mathrm{AE}}$, is defined as the time gap between stimulation to the input neuron and its corresponding stimulation to the output neuron (and similarly for other neuronal chains composing the DLG). Consequently, the time gap between two stimulations of the output neuron is $\left|\tau_{\mathrm{AE}}-\tau_{\mathrm{BE}}\right|$. Initially, $\tau_{\mathrm{AE}}$ is shorter in comparison to the one-neuron chain,
$\tau_{\mathrm{BE}}$. This ratio reverses as repeated simultaneous stimulations are given to the input neurons, $\mathrm{A}$ and $\mathrm{B}$, and the neuronal response latencies increase (Figure 2B; Vardi et al., 2013b). For each input stimulation Figure 2B (upper panel, Vardi et al., 2013b) presents the time-lag between the two weak stimulations of neuron $\mathrm{E}$, $\left|\tau_{\mathrm{AE}}-\tau_{\mathrm{BE}}\right|$, as well as whether a spike was evoked from neuron E. For a time-lag $\left|\tau_{\mathrm{AE}}-\tau_{\mathrm{BE}}\right|$ larger than $\sim 0.5 \mathrm{~ms}$ (varies among different neurons and stimulation parameters) the output neuron (E) does not respond, independent of the input stimulation, indicating a "NULL" operating mode of the logic-gate. In the intermediate region, $\left|\tau_{\mathrm{AE}}-\tau_{\mathrm{BE}}\right|$ smaller than $\sim 0.5 \mathrm{~ms}$, the input/output interrelations typically follow that of an AND-gate. Hence, this neuronal gate exhibits NULL-AND-NULL dynamic logic transitions (Table 1, 1st row).

At the bottom of Figure 2B (Vardi et al., 2013b) different segments of the voltage recordings of neuron $\mathrm{E}$ are displayed, the colored (green, orange) lines are the stimulations arriving from the input chains $\left(\tau_{\mathrm{AE}}, \tau_{\mathrm{BE}}\right.$, respectively). Initially, $\tau_{\mathrm{AE}}$ is shorter than $\tau_{\mathrm{BE}}$ (left recording) thus the "green" stimulation arrives at the output neuron before the "orange" one. This order is reversed later (right recording). The second and third recordings demonstrate the AND region; in the second recording two weak stimulations arriving at neuron $\mathrm{E}$ result in an evoked spike. In the case of response failure of one of the neurons comprising the left input chain (third recording), neuron E receives only one weak stimulation from neuron B and therefore does not fire,

\begin{tabular}{|c|c|c|c|c|}
\hline \multirow[t]{2}{*}{ Logic-gate } & \multicolumn{3}{|c|}{ Truth table } & \multirow[t]{2}{*}{ Dynamic logic operation } \\
\hline & $\mathrm{in}_{1}$ & $\mathrm{in}_{2}$ & output & \\
\hline \multirow[t]{4}{*}{ AND } & 0 & 0 & 0 & NULL $\rightarrow$ AND $\rightarrow$ NULL \\
\hline & 0 & 1 & 0 & \\
\hline & 1 & 0 & 0 & \\
\hline & 1 & 1 & 1 & \\
\hline \multirow[t]{4}{*}{ OR } & 0 & 0 & 0 & $I F\left[i_{1}\right]+I F\left[i_{2}\right] \rightarrow O R \rightarrow I F\left[i_{2}\right]+I F\left[i_{1}\right]$ \\
\hline & 0 & 1 & 1 & \\
\hline & 1 & 0 & 1 & \\
\hline & 1 & 1 & 1 & \\
\hline \multirow[t]{2}{*}{ NOT } & 0 & & 1 & $1 \rightarrow \mathrm{NOT} \rightarrow 1$ \\
\hline & 1 & & 0 & \\
\hline \multirow[t]{4}{*}{ XOR } & 0 & 0 & 0 & $\mathrm{OR} \rightarrow \mathrm{XOR} \rightarrow \mathrm{OR}$ \\
\hline & 0 & 1 & 1 & \\
\hline & 1 & 0 & 1 & \\
\hline & 1 & 1 & 0 & \\
\hline
\end{tabular}

The first column lists the logic-gates. The second column details the truth table, the input/output relations. The third column presents the confirmed dynamic transitions among different logic operating modes, as a gate was repeatedly stimulated. The symbols "0/1" stand for a non-evoked/evoked spike, "NULL" indicates a non-evoked output spike independent of the inputs and IF(in $\left.{ }_{i}\right)$ indicates an output identical to the ${ }^{\text {th }}$ input. The order of IF(in $\left.)_{1}\right)$ and IF(in $\left.{ }_{2}\right)$ in the second row indicates the timing of their effects on the output unit. Reproduced upon permission by Vardi et al. (2013b). 
in agreement with the logic operation of an AND-gate (Table 1, 1st row).

The experimental results also indicate a slight asymmetry, where the first NULL-AND transition occurs at a shorter time-lag in comparison to the second AND-NULL transition (Figure 2B; Vardi et al., 2013b). This asymmetry might be attributed to the stretching of the response latency of neuron $\mathrm{E}$ in between the two transitions.

\section{DYNAMIC OR-GATE}

The experimental setup of the dynamic OR-gate is similar to the AND-gate (Figure 2A; Vardi et al., 2013b), however all the stimulations are now strong (Figure 3A; Vardi et al., 2013b) and are individually capable of reliably generating an evoked spike.
The output neuron, F, generates two evoked spikes when the time-lag between the two incoming stimulations is large enough (compared to the refractory period), typically greater than $4 \mathrm{~ms}$ (Figure 3B; Vardi et al., 2013b). To enhance the dynamic range of time-lags between two stimulations to neuron $F$, the gate now consists of six neurons in total and a four-neuron input chain (Figure 3A; Vardi et al., 2013b). Consequently, the relative stretching of the two input neuronal chains, $\left|\tau_{\mathrm{AF}}-\tau_{\mathrm{BF}}\right|$ exceeds $\sim 5$ ms (Figures 3C,D; Vardi et al., 2013b).

The dynamic logic operating modes are exemplified for an entry from a region of typically two evoked spikes (when both input neurons are stimulated) into an OR mode, characterized by a single output spike in response to stimulation in in $_{1} O R$ in $_{2}$ (Figure 3C; Vardi et al., 2013b), and for an exit from an OR
A

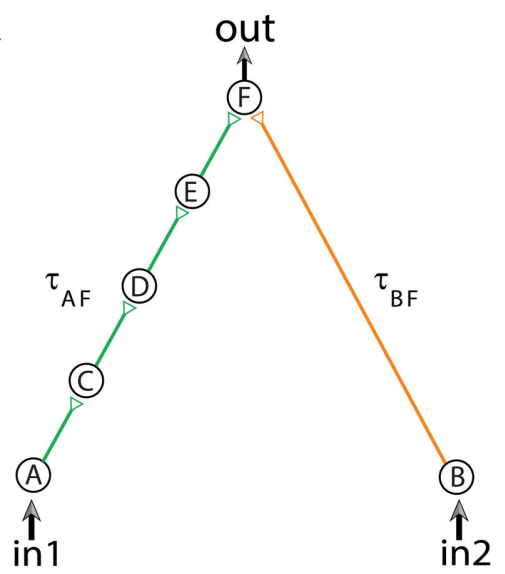

C

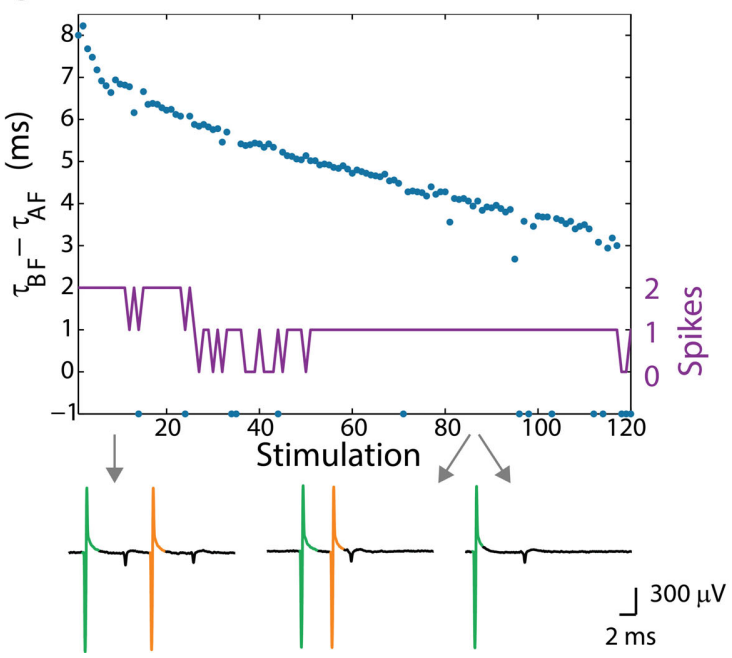

FIGURE 3 | (Color online) Dynamic OR gate. (A) Schematic of an OR-gate consisting of a four-neuron input chain (green) and a one-neuron input chain (orange), where all stimulations are strong. (B) Independent experiments for a fixed time-lag $\tau_{\mathrm{AF}}-\tau_{\mathrm{BF}}$. The probability for neuron $\mathrm{F}$ to respond by two-spikes was averaged over several tens of input stimulations. (C) Input stimulations at a rate of $10 \mathrm{~Hz}$ resulting in dynamic changes of $\tau_{\mathrm{BF}}-\tau_{\mathrm{AF}}$ from 8 to $3 \mathrm{~ms}$ (blue dots). A dynamic transition from the region of typically two output spikes to an OR operating mode

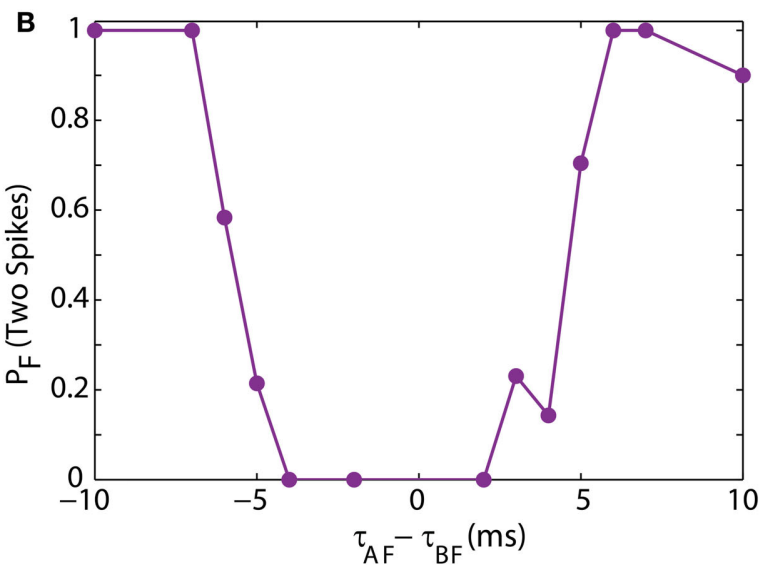

D

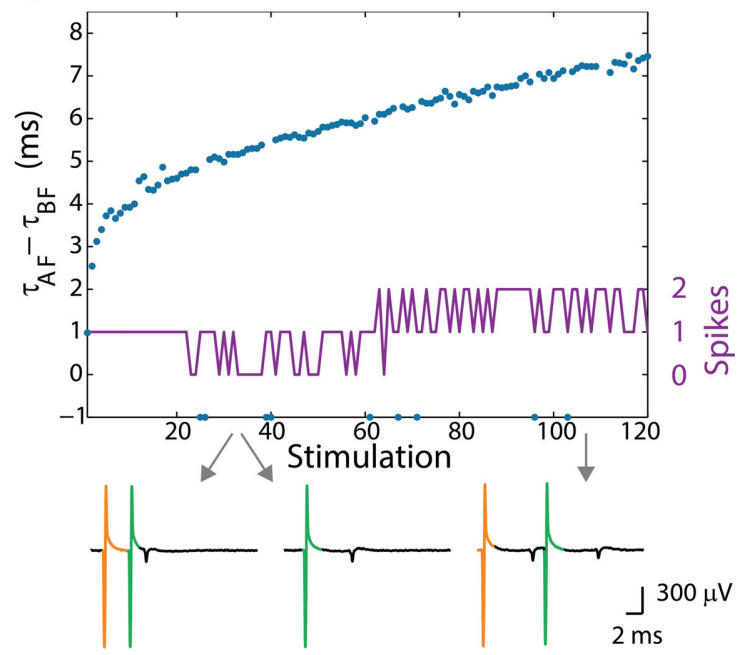

(similar to the entry in B) occurs after $\sim 30$ input stimulations. Missed evoked spikes resulting in only one stimulation to neuron $F$ are marked as "-1." (D) Similar to the entry in (B), $\tau_{\mathrm{AF}}--\tau_{\mathrm{BF}}$ increases from $\sim 2.5$ to $7 \mathrm{~ms}$ (blue dots) and a dynamic exit from the OR region to the region of typically two evoked spikes occurs after $\sim 60$ input stimulations. Different segments of the voltage recording of neuron $F$ are exemplified below, the arrows point from different scenarios to their matching recordings. Reproduced upon permission from Vardi et al. (2013b). 

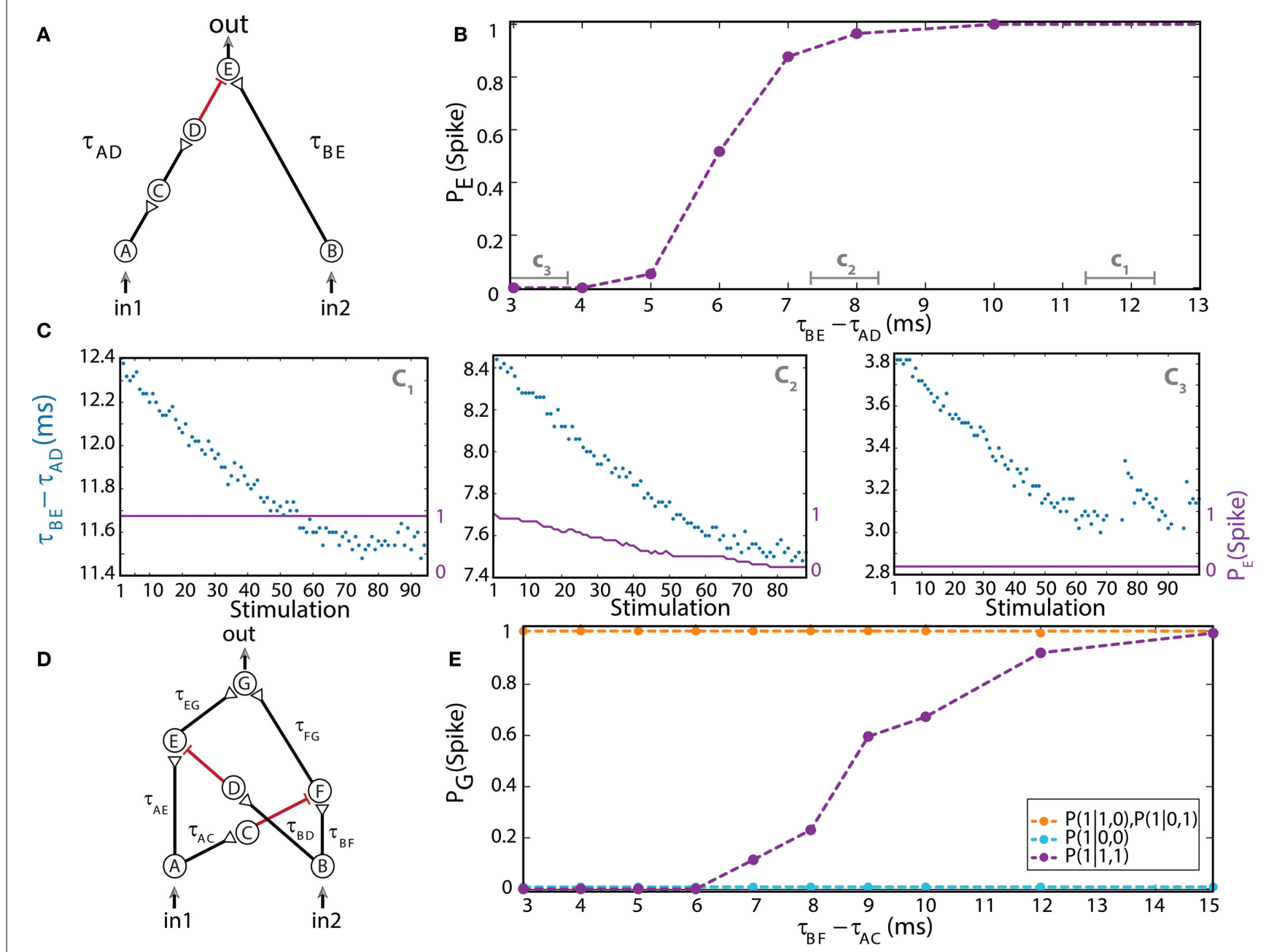

FIGURE 4 | (Color online) Dynamic NOT and XOR gates. (A) Schematic of a NOT-gate consisting of five neurons, with one inhibition (red). A

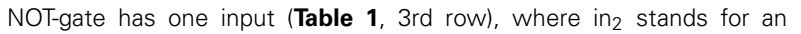
outer stimulation which is given for every computation. (B) Independent experiments for a fixed time-lag $\tau_{\mathrm{BE}}-\tau_{\mathrm{AD}}$ and $\tau_{\mathrm{BE}}=80 \mathrm{~ms}$. The input neurons are simultaneously stimulated at $1 \mathrm{~Hz}$. (C) Input stimulations at a rate of $10 \mathrm{~Hz}$ resulting in dynamic changes in $\tau_{\mathrm{BE}}-\tau_{\mathrm{AD}}$, averaged over a sliding window of 20 stimulations, as shown by time segments $C_{1}, C_{2}$, and $c_{3}$ in (B). (D) Schematic of a XOR-gate containing two inhibitory stimulations (red). (E) Input neurons are simultaneously stimulated at $1 \mathrm{~Hz}$. Independent experiments where $\tau_{\mathrm{BF}}-\tau_{\mathrm{AC}}$ is varied, a fixed time-lag $\tau_{\mathrm{AE}}-\tau_{\mathrm{BD}}=3 \mathrm{~ms}$ was selected to inhibit the stimulation from neuron $\mathrm{A}$, $\tau_{\mathrm{AE}} \approx 100, \tau_{\mathrm{BF}} \approx 50$, and $\tau_{\mathrm{AG}} \approx \tau_{\mathrm{BG}}=150 \mathrm{~ms}$ were performed (circles connected with dashed guideline). The conditional probabilities of an evoked spike of the output neuron $G$ are presented by the three colored dashed lines. Reproduced upon permission from Vardi et al. (2013b). mode (Figure 3D; Vardi et al., 2013b). In the entry to the OR operating mode, the stimulation from neuron A (green) arrives prior to the stimulation from neuron B (orange), whereas in the exit, the "orange" stimulation arrives prior to the "green" one, and accordingly the order of the logic operations is presented in Table 1, 2nd row. Note that $O R$ represents one logic operation with one possible evoked spike, whereas the response of the DLG at the beginning/end is composed of 2 consecutive temporally independent logic operations. This can also be seen in the voltage recordings of neuron F, Figures 3C,D (Vardi et al., 2013b).

\section{DYNAMIC NOT-GATE}

The implementation of the dynamic NOT-gate is similar to the previous ones (Figures 2A, 3A; Vardi et al., 2013b), however it contains an inhibitory stimulation from neuron $\mathrm{D}$ to $\mathrm{E}$
(Figure 4A; Vardi et al., 2013b). It inhibits, for a limited time interval, the response of neuron $\mathrm{E}$ to an excitatory stimulation arriving from neuron B. Note that a typical NOT-gate consists of a single input (Table 1, 3rd row), thus in our case the "con-

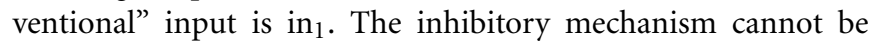
achieved by shaping the stimulation's amplitude or its sign. The use of a different cocktail of synaptic blockers, mainly suppressing the excitatory synapses (Supplementary Material), enables the implementation of inhibitory stimulations, as discussed in Vardi et al. (2013b). Since the effect of an inhibitory stimulation is measurable only in the presence of an excitatory stimulation, we apply an outer stimulation (indicated as $i_{2}$ in Figure 4A; Vardi et al., 2013b) in the spirit of electronic circuits. This outer excitatory stimulation is applied each time a computation is requested, simultaneous with the stimulation of $\mathrm{in}_{1}$. 
For low stimulation rates, the stretching of neuronal response latencies is negligible; hence the logic operation of the gate was independently measured for each relative delay between excitation and inhibition of the output neuron $\mathrm{E}, \tau_{\mathrm{BE}}-\tau_{\mathrm{AD}}$ (Figure 4B; Vardi et al., 2013b) under low stimulation rate. When the inhibitory stimulation is given $5 \mathrm{~ms}$ or less prior to an excitatory stimulation - the inhibition is almost absolute. This effect deteriorates for larger time gaps, until it vanishes around $10 \mathrm{~ms}$ (Figure 4B; Vardi et al., 2013b). For high stimulation rates, a dynamic behavior of the logic operation is demonstrated, where a relatively sharp transition is observed from a reliable relay of an arriving stimulation to an absolute blocker, a NOT-gate (Figure 4C; Vardi et al., 2013b). In a reversed order, it is evident that an excitation sufficiently prior to inhibition is effective. However, it was experimentally difficult to locate this transition, since the spike detection is disrupted by the artifact of the inhibitory stimulation. Nevertheless, for an inhibition chain consisting of a larger number of neurons, consecutive 1-NOT-1 logic operating modes are anticipated in a single experiment (Table 1, 3rd row).

\section{DYNAMIC XOR-GATE}

The logic operation of a XOR-gate is identical to an OR-gate, except for the entry $(1,1)$, two input stimulations, which do not generate an evoked spike (Table 1, 4th row). Its implementation is similar to the OR-gate setup with additional two inhibitory stimulations (Supplementary Material), from the first input to a neuron belonging to the chain of the second input and vice versa (red connections in Figure 4D; Vardi et al., 2013b). For low stimulation rates, the neuronal response latencies remain unaffected and the logical operation of the XOR-gate was tested independently for each relative delay between excitation and inhibition, $\tau_{\mathrm{BF}}-\tau_{\mathrm{AC}}$ (Figure 4E; Vardi et al., 2013b). The delays $\tau_{\mathrm{AE}}$ and $\tau_{\mathrm{BD}}$ were selected such that the inhibition to neuron $\mathrm{E}$ is effective and consequently a transition from XOR to OR operating modes is exemplified (Figure 4E; Vardi et al., 2013b). The confirmation of this dynamic logic operating transitions, however, requires much longer neuronal chains and is examined in section Theoretical Analysis using an analytical approach.

\section{THEORETICAL ANALYSIS}

Complex DLGs based on time-dependent neuronal response latencies usually require larger scale networks consisting of a greater amount of neurons. Their experimental implementations are associated with some difficulties, especially when delays, timing of stimulations and evoked spikes must be monitored on sub-millisecond timescales. Hence, the computational horizon of the new logic-gates requires a simplified theoretical framework which is based on the following two assumptions.

First, for each neuron comprising the gate, we assume a constant increase in the neuronal response latency per evoked spike, $\Delta$, independent of its current latency and identical for all neurons. This assumption approximately fits the second state of the latency increase (stimulation responses 100-650 in Figure 1A; Vardi et al., 2013b). Under this assumption the latency of a neuron can be written as:

$$
l(q)=l_{0}+q \triangle
$$

where $l_{0}$ stands for the neuron's initial response latency, $q$ is the number of evoked spikes and $\Delta$ is a constant which in our experiments is typically in the range of $2-7 \mu$ s. Similarly, the time delay of a chain is defined as the time-lag between the stimulation of the first neuron and the stimulation of the neuron at the end of the chain. Consequently the time delay for a chain consisting of $n$ neurons is given by

$$
\tau(q)=\tau_{0}+n q \triangle
$$

where $\tau_{0}$ stands for the initial time delay of the chain. Similar to the experimental results, the increase in the delay of a chain is linear with the number of neurons in the chain, $n$.

The second assumption is that a strong excitatory stimulation generates an evoked spike with a probability of 1 (1:1 response), thus the number of evoked spikes of a neuron is equal to the number of its stimulations.

\section{DYNAMIC AND-GATE}

The AND-gate is examined below under this theoretical framework and results are compared to the experimental findings (section Experimentally Examined DLGs, Figure 2A; Vardi et al., 2013b).

The delays of the green and orange chains (Figure 2A; Vardi et al., 2013b) as a function of the stimulation number are presented in Figure 5A using equation (2) with $\Delta=$

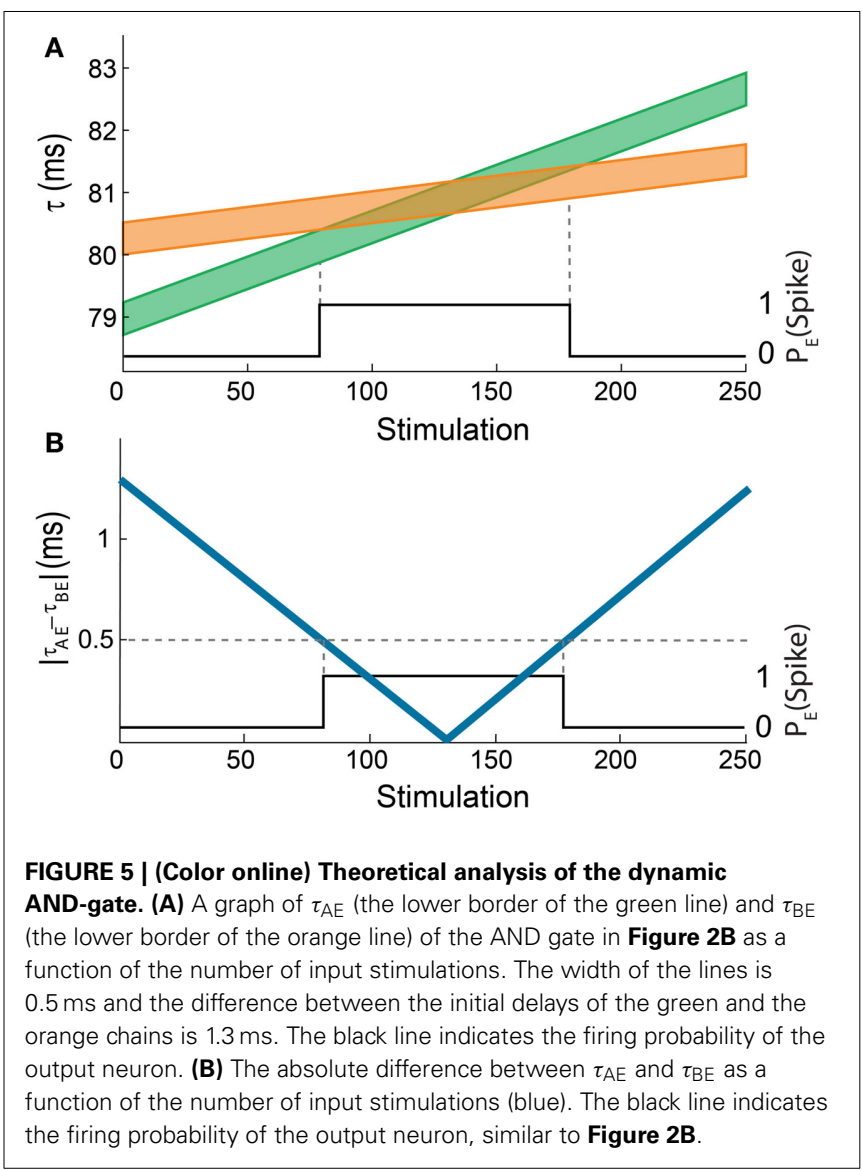


$5 \mathrm{~ms}$. The broadening of each line by $0.5 \mathrm{~ms}$ represents the maximal time delay between two stimulations of neuron $\mathrm{E}$, $\left|\tau_{\mathrm{AE}}-\tau_{\mathrm{BE}}\right|$, which generates an evoked spike. Hence, the intersection between these two lines represents the region where neuron $\mathrm{E}$ fires. In agreement with the experimental results, the initial delay of the green chain (neurons A, $\mathrm{C}$, and D) is shorter than the delay of the orange chain (neuron B).

The similarities between the dynamical transition predicted by the theoretical model (Figure 5B) and the experimental results (Figure 2B; Vardi et al., 2013b) are evident. Obviously, there are some minor differences; however the qualitative behavior is the same. This validation of the theoretical model supports its applicability for complex DLGs which are at the moment beyond experimental realization.

\section{GENERALIZED AND-GATE}

Using the theoretical model presented above, several DLGs are examined. These DLGs implement complex transitions illustrating additional properties of their dynamics. To simplify the presentation we mainly concentrate on generalized AND-gates.

The first examined generalized AND-gate consists of three excitatory input chains consisting of $1 / 2 / 5$ neurons (Figure 6A). A dashed arrow stands for a weak stimulation such that at least two weak stimulations at a time-lag less than $0.4 \mathrm{~ms}$ are required to generate an evoked spike in the output neuron. The initial time delays from the stimulations of the three input neurons to the stimulation of the output neuron are selected to be $30 / 27 / 25 \mathrm{~ms}$ for the chains consisting of 1/2/5 neurons, respectively. Note that in the limiting case of simultaneous stimulation to the three input neurons, this complex DLG is equivalent to the DLG consisting of only two input signals but with a more structured internal wiring, as exemplified in Figure 6B. Using equation (2) with $\Delta=$ $0.004 \mu \mathrm{s}$ ( $4 \mathrm{~ms}$ ) we show the time delays of the three input chains as a function of the number of given stimulations in Figure 6C. An intersection of two lines implies that the difference of the matching delays is less than $0.4 \mathrm{~ms}$, thus resulting in a spike of the output neuron (black line in Figure 6C). In the intersection regions the gate acts as an AND gate for the two appropriate inputs (e.g., in the intersection of the "blue" and "orange" lines the output neuron fires if and only if in ${ }_{1} A N D$ in $_{2}$ are stimulated).

Increasing the input stimulation rate typically results in an enhanced stretching of the neuronal latency per spike (Vardi et al., 2012a). Results for $\Delta=0.006 \mathrm{~ms}(6 \mathrm{~ms})$ are presented in Figure 6D, where it is noticeable that the gate dynamics still consists of three entries to AND-regions. Moreover, the firing regions of Figures $6 \mathrm{C}, \mathrm{D}$ are the same under the rescaling of the stimulation axis by $0.004 / 0.006$. Hence, we conclude that the dynamic transitions are robust to different stimulation frequencies. Nevertheless, it is clear that different initial delays to the three chains can reduce the three AND-reentries to two, one, or even remove the entire AND operation (e.g., the initial purple chain's delay is greater than the initial blue chain's delay which is greater than the initial orange chain's delay). Another important factor is the relative number of neurons comprising the neuronal chains. For illustration, in the case that the purple chain is reduced from five neurons (Figure 6A) to three, the three AND-regions merge into one region (Figure 6E).

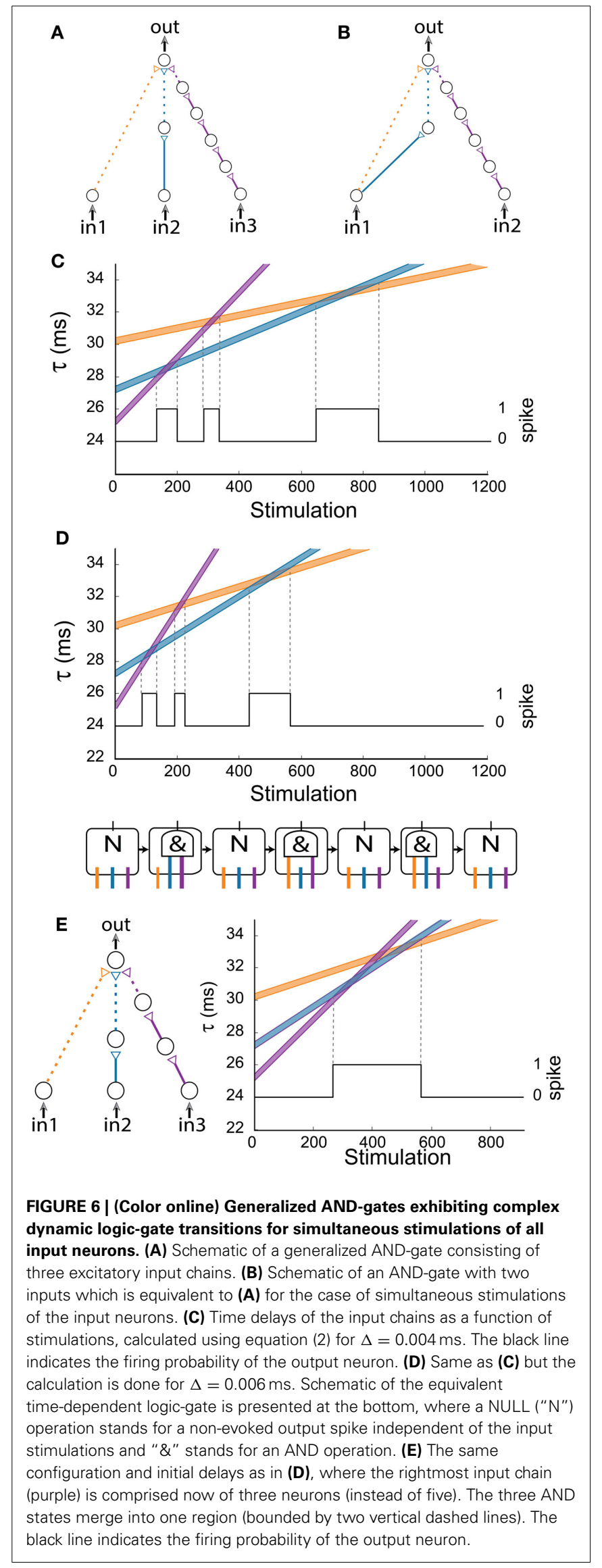



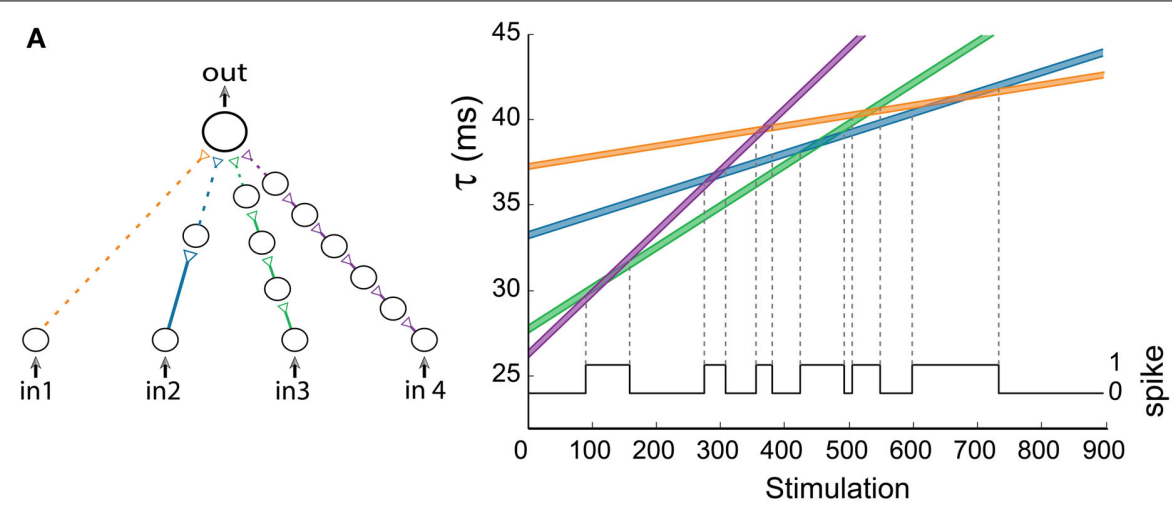

B
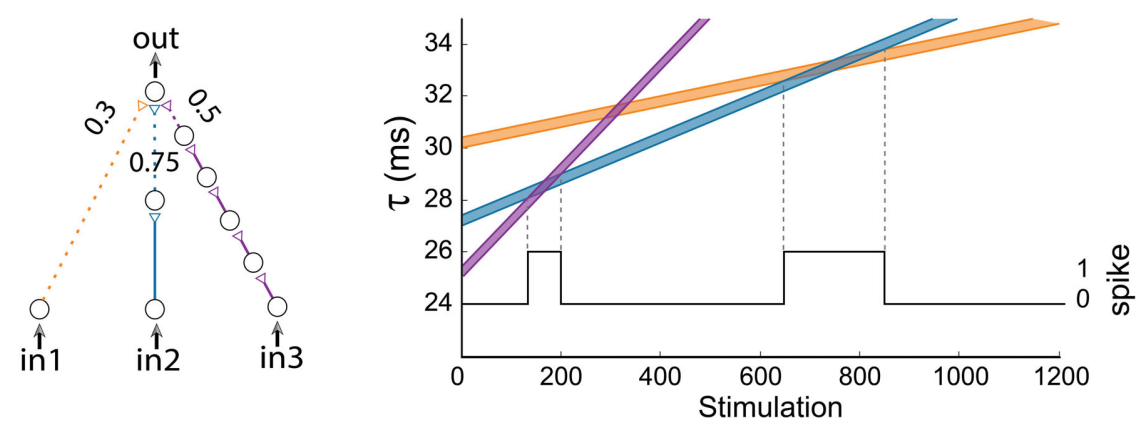

FIGURE 7 | (Color online) Advanced logic-gates. (A) An AND-gate consisting of four inputs. The time delays of the input chains are presented as a function of the number of stimulations, calculated using equation (2) for $\Delta=0.006 \mathrm{~ms}$. The black line indicates the firing probability of the output neuron. (B) An AND-gate of the same architecture as in
Figure $\mathbf{6 A}$, but the three weak stimulations have different strengths. The time delays of the input chains are presented as a function of the number of stimulations, calculated using equation (2) for

$\Delta=0.004 \mathrm{~ms}$. The black line indicates the firing probability of the output neuron.
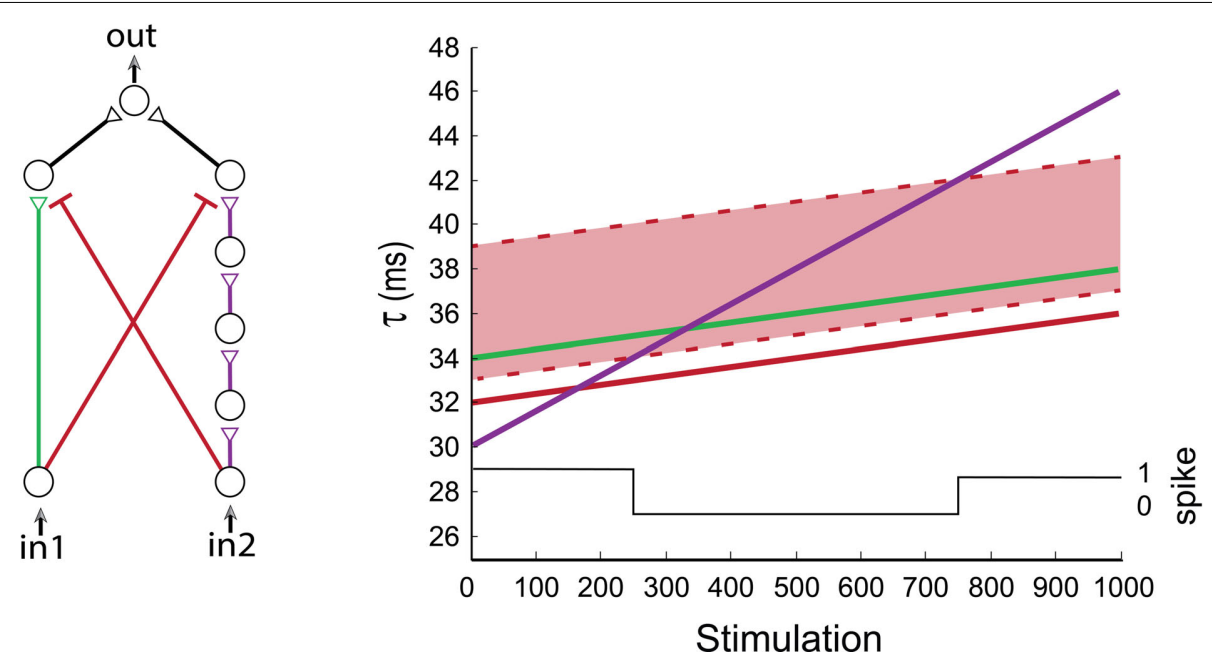

FIGURE 8 | (Color online) Dynamic XOR gate. A dynamic XOR-gate with $2 / 5$ neuronal excitatory input chains (green/purple), and two inhibitory stimulations (red) with identical initial delays of $32 \mathrm{~ms}$. The inhibition is effective in a time window of $[1,7] \mathrm{ms}$ prior to the excitatory stimulation and is represented by the light-red region. The first input is always blocked (as the green line is always inside the light-red region). The black line indicates the firing probability of the output neuron. A temporal XOR operating mode is observed at the stimulation range of [250, 750], where simultaneous stimulations (of $\mathrm{in}_{1}$ and $\mathrm{in}_{2}$ ) result in no evoked spikes of the output neuron.
In a more general scenario of $\mathrm{k}$ input chains to the output neuron where all input neurons are simultaneously stimulated, the maximal number of AND regions scales quadratically with $k$, since the number of intersections of $k$ non-parallel lines is
$0.5 \mathrm{k}(k-1)$. To exemplify a scenario where the number of transitions exceeds $\mathrm{k}$, a gate with $k=4$ with $1 / 2 / 4 / 6$ neuronal chains is examined (Figure 7A). Using equation (2) with $\Delta=0.006 \mathrm{~ms}$, where the maximal time-lag between two weak stimulations 

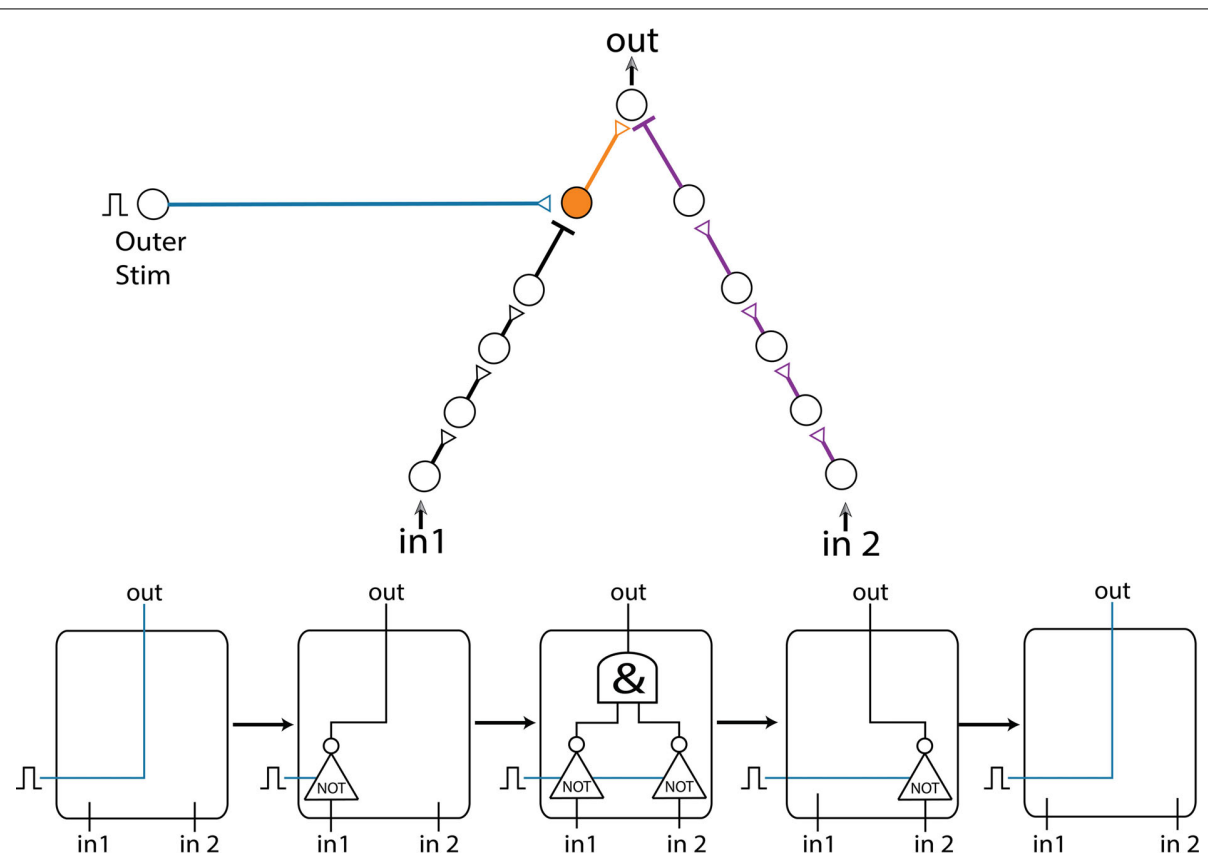

FIGURE 9 | (Color online) Multiple operation modes. A gate consisting of two inputs, an outer stimulation and two inhibition chains (black and purple), exemplifying transitions among 5 different operation modes. The increase of the delays results in a transition between the logic operation modes illustrated by the flow chart at the bottom.
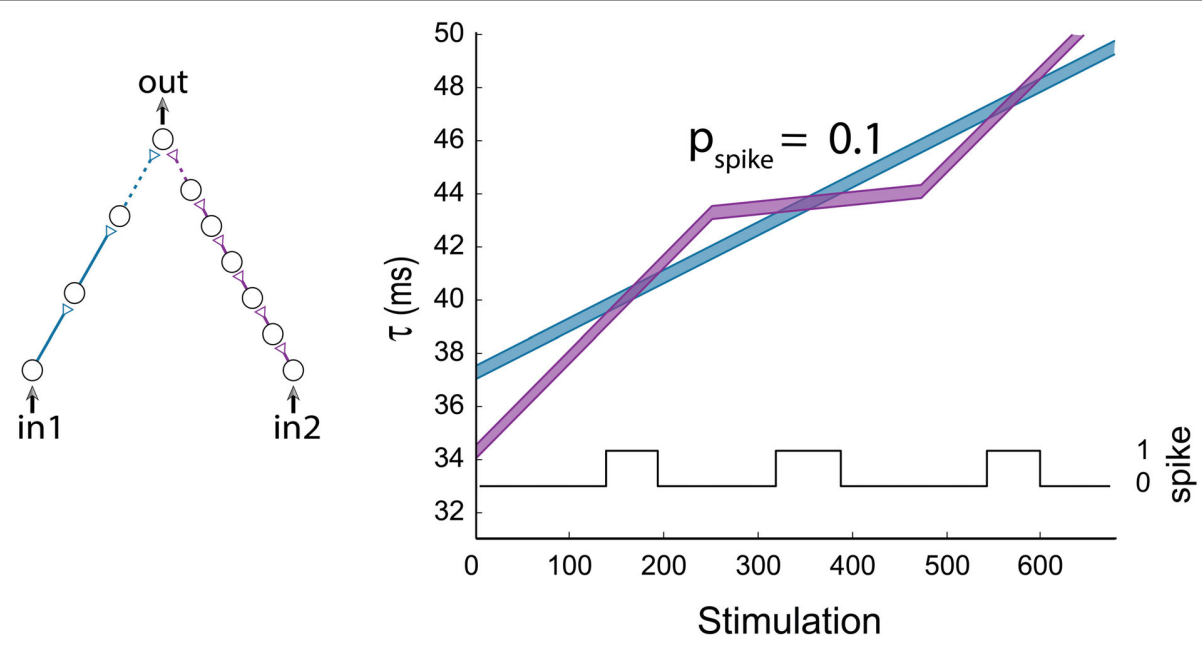

FIGURE 10 | (Color online) Non periodic input stimulations. An AND-gate with the following input pattern: $i_{1}$ is stimulated at a fixed rate, while the stimulation of $\mathrm{in}_{2}$ is relatively moderated in the stimulation period $(250,475)$ to probability 0.1 in comparison to in ${ }_{1}$. The horizontal axis stands for the number of stimulation given to $\mathrm{in}_{1}$. The black line indicates the firing probability of the output neuron per stimulation to $\mathrm{in}_{2}$. resulting in an evoked spike is $0.4 \mathrm{~ms}$, one can spot six $\left(0.5^{*} 4^{*} 3=\right.$ 6) transitions to an AND operating mode (Figure 7A).

To illustrate how the strength of the connections between neurons affects the gate's transitions, we examine an AND-gate of the same architecture as in Figure 6A, but the three input stimulations to the output neuron are weak and have the relative strengths of $0.3 / 0.75 / 0.5$ for the orange/blue/purple connections, respectively (Figure 7B). To generate a spike at the output neuron, the sum of the stimulation strengths must exceed a threshold of 1 .
Note that the second transition to an AND-gate (Figure 6A) disappears (Figure 7B), since the sum of the strengths of the orange connection and purple connection is 0.8 and does not exceed the threshold (Markram and Tsodyks, 1996).

\section{DYNAMIC XOR-GATE}

The temporal activation of the XOR-gate was experimentally exemplified by a series of independent setups, where one of the inhibitory delays was gradually updated (Figure 4E). To illustrate 
the transitions of the dynamic XOR operation modes, three neurons are added to the excitatory purple input chain (Figure 8) in comparison to the experimental setup (Figure 4D). Initially we set the same delay for both inhibitions which are effective in a time window of $[1,7] \mathrm{ms}$ prior to the excitatory stimulation (i.e., if an inhibitory stimulation occurs at time $\mathrm{T}$ then the neuron will not respond to any stimulation in the time interval [ $\mathrm{T}$ $+1, \mathrm{~T}+7] \mathrm{ms})$. The region where the excitatory stimulation is inhibited is depicted by the light-red region bounded by dashed red lines (Figure 8). Consequently, $\mathrm{in}_{1}$ is always inhibited by $\mathrm{in}_{2}$, while $\mathrm{in}_{2}$ is only temporarily inhibited by $\mathrm{in}_{1}$, and a temporal $\mathrm{XOR}$ operation is observed.

\section{TRANSITION AMONG MULTIPLE MODES}

In the following example we present a gate consisting of two inputs and an outer stimulation given for every computation (as in section Dynamic NOT-Gate, NOT-Gate), resulting in four different logic operating modes (Figure 9). The gate contains two inhibition chains (black and purple), with initial time delays of 30 and $42 \mathrm{~ms}$, respectively. Both inhibitions are effective in a time window of $[1,7] \mathrm{ms}$ prior to an excitatory stimulation (as in section Dynamic XOR-Gate). The initial blue and orange delays are 40 and $10 \mathrm{~ms}$, respectively. For every computation of the logicgate, the outer stimulation and the stimulations of the input neurons are given simultaneously. In the initial stage, the output neuron fires as a result of the outer stimulation independent of both inputs. The inhibition is ineffective, since the delays of the black and purple chains are too short (in comparison to the blue and orange delays). The black and purple delays increase with the neuronal response latencies, and the gate enters its second operating mode. The entire delay of the black chain grows relatively faster than the delay from the outer stimulation (blue) due to the number of neurons comprising each chain. Hence, when stimulated repeatedly, the delay of the black chain increases enough to inhibit the output spike which is caused by the outer stimulation, whereas the delay of the purple chain is still too short to affect the output. Consequently, the output spike caused by the excitatory outer stimulation is inhibited by $\mathrm{in}_{1}=1$, resulting in a $\operatorname{NOT}\left(\mathrm{in}_{1}\right)$ functionality. In the third operation mode, the delays of the black and purple chains are both long enough to cause inhibition, therefore an output evoked spike will occur only in the case where both inputs are 0 . In the fourth operation mode, the inhibition caused by the purple chain is still effective, whereas the inhibition caused by the black chain vanishes as a result of its enhanced stretching, resulting in a $\operatorname{NOT}\left(\mathrm{in}_{2}\right)$ functionality. In the final operation mode, the delays of both inhibition chains are too large to inhibit the output spike caused by the outer stimulation, thus the logic-gate returns to its initial functionality where an output spike is generated independent of both inputs.

\section{VARYING INPUTS}

So far, the limited case where simultaneous stimulations were given to all inputs of the gates was discussed. This scenario revealed many properties of the DLGs, however it is clear that more structured types of temporal input stimulations are expected to enrich the dynamic transitions. To exemplify this scenario we consider an AND-gate with two input chains consisting of three and six neurons (Figure 10). Applying a fixed stimulation rate to the two input neurons results solely in one AND-region (first AND region in Figure 10). A temporal reduction in the probability for a stimulation of the purple input chain results in a moderated latency increase, thus the delay of the blue chain becomes larger than the delay of the purple chain, and a second AND region emerges. When a fixed stimulation rate is applied again to the two input neurons, the delay of the purple chain overshoots the delay of the blue one, resulting in a reentry to a third AND region (Figure 10).

\section{MULTIPLE COMPONENT NETWORKS AND SIGNAL PROCESSING}

We differentiate between two main computational capabilities of the DLGs. The first approach aims at reaching a specific operating mode of the dynamic gates using intentional repeated stimulations, which enables the desirable computations on occasional inputs. In the second approach, we are not interested in performing computations using specific logic operations but rather in using the dynamic properties of the gates. The purpose is to discover information regarding the input sequences. This approach is exemplified by a collaboration of a large number of dynamic components which together can implement a basic edge detector (Figure 11).

The input of an edge detector is a vector of size $n$ and its task is to identify radical structural changes or discontinuities. For instance, if the vector's values represent a degree of brightness as a function of (one dimensional) position, the mission of an edge detector is to identify two consecutive points with significant changes in their brightness. The proposed edge detector, consisting of $n$ input neurons, is sketched in Figure 11. Each two consecutive neurons serve as inputs to a dynamic AND-gate. Initially all delays are equal, thus simultaneous stimulations to all input neurons result in the firing of all output neurons. We assume that the number of stimulations of each input neuron is proportional to the brightness of the corresponding position in the input vector. To avoid extreme scenarios we assume that the inter-spike-intervals of each neuron do not vary much in time. Since the stretching of each delay is proportional to the number of input stimulations, a significant difference between two input chains of a dynamic AND-gate will be developed in case of a significant change between the brightness of two consecutive inputs. As a result their shared dynamic AND-gate will reach a NULL

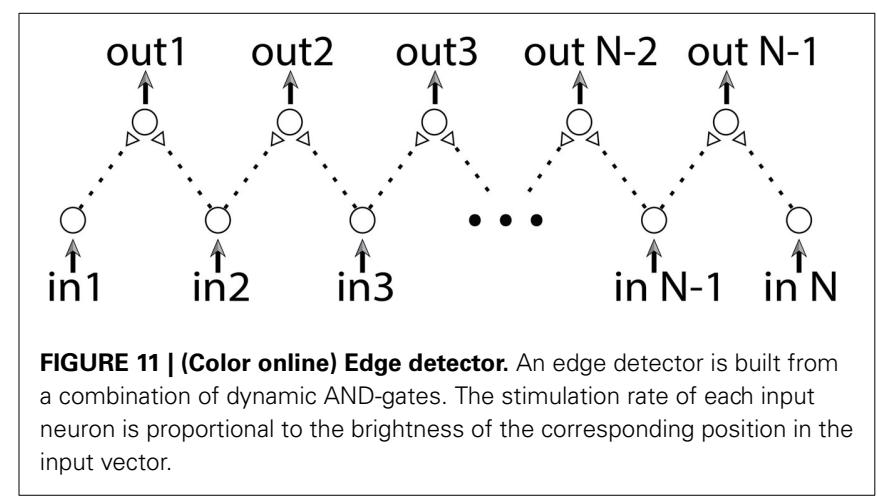


A

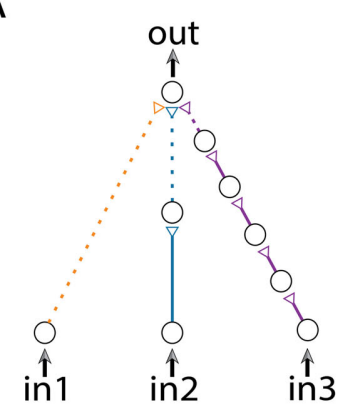

B

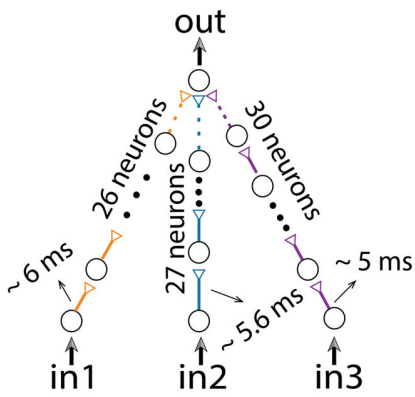

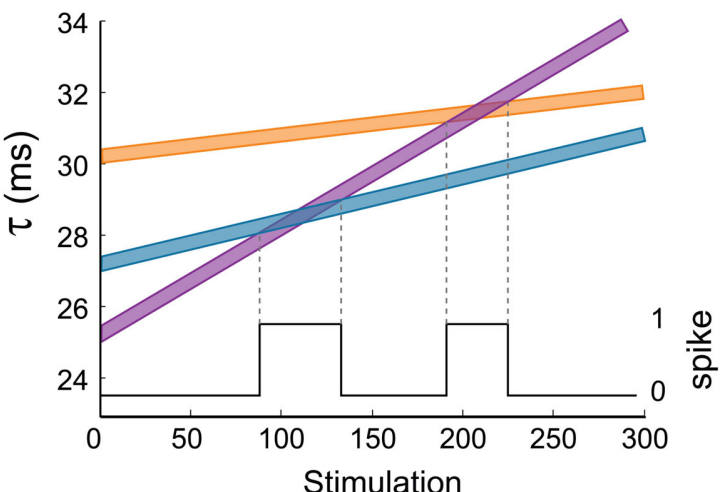

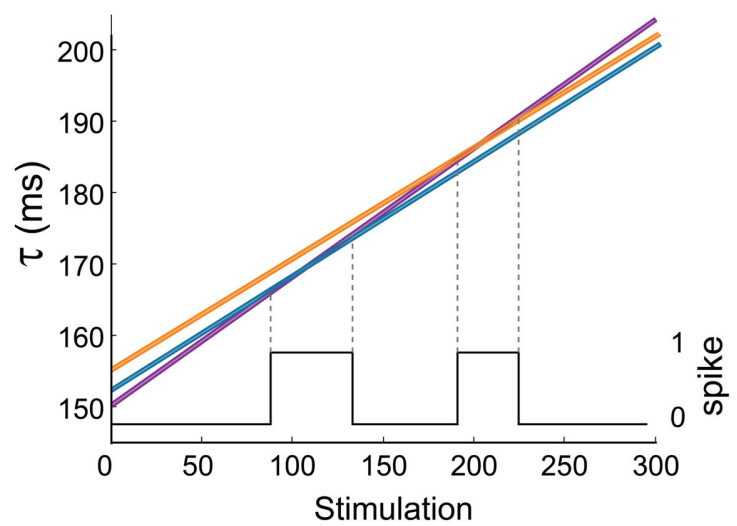

FIGURE 12 | (Color online) Short delays. (A) The dynamic AND-gate and a portion of the graph presented in Figure 6, showing 2 transitions to AND regions. This gate consists of three input chains of $1 / 2 / 5$ neurons each, and contains relatively long delays, up to $34 \mathrm{~ms}$. (B) A similar AND-gate with long chains consisting of 26/27/30 neurons, resulting in short delays of 5-6 ms between consecutive neurons. For $\Delta=0.006 \mathrm{~ms}$ the delays of the input chains are presented in the right graph as a function of stimulation number, where the black line indicates the firing probability of the output neuron. state. The examination of edges will be then achieved by a simultaneous stimulation to all input neurons. The sensitivity of the detection is determined by the duration of the stimulating period of the input neurons, where longer periods result in higher sensitivity. Since the stretching of the neuronal response latency is reversible, this edge detector can be reused after a short period without input stimulations.

\section{SUITABILITY OF DYNAMIC LOGIC-GATES TO BRAIN FUNCTIONALITY}

It is implausible to assume that brain functionality is as simple as a combination of standard SLGs, especially since it requires accurate predefined set of delays that are static and do not change over time. In this study we introduced a paradigm which is more suitable for brain functionality, DLGs. We will now discuss the feasibility and reliability of the DLGs in an environment more suitable for the functioning brain.

\section{SHORT SYNAPTIC DELAYS}

Our experimental procedure, corroborated and extended by theoretical evidence, was examined under conditions of synaptic delays of a few tens of millisecond, which are typically beyond cortical synaptic delays. This constraint can be adapted to the time scales of synaptic delays and transient periods of the brain, several ms (Abeles, 1991). From a theoretical point of view, the functionality of the proposed feedforward logic-gates is a function of the relative difference between the stretching of the input chains, regardless of the absolute number of neurons constituting each chain. Therefore, all synaptic delays can be shortened to the order of a few milliseconds using long synfire chains (Abeles, 1991; Abeles et al., 2004; Ikegaya et al., 2004; Izhikevich, 2006; Pastalkova et al., 2008; Long et al., 2010). For illustration, let us concentrate on Figure 12A consisting of relatively long delays, up to $34 \mathrm{~ms}$. A similar modified dynamic gate consisting of long synfire chains, of 26/27/30 neurons (Figure 12B), resulting in 5$6 \mathrm{~ms}$ delays between consecutive neurons (including the neuronal response latency). Note that the relative difference between the amount of neuronal populations comprising the input synfire chains remain the same as in Figure 12A, i.e., $2-1=27-26$ and 5$1=30-26$. Therefore, both AND-gates have identical transition timings between NULL and AND logic operations.

\section{TIME SCALES OF OPERATION MODES}

The reported periods of operating logic modes consist of a few hundred stimulations, which exceed a few seconds under stimulation frequencies that are in the order of few dozens. These periods can be shortened by two orders of magnitude using the following two enhanced stretching effects: Long synfire chains 

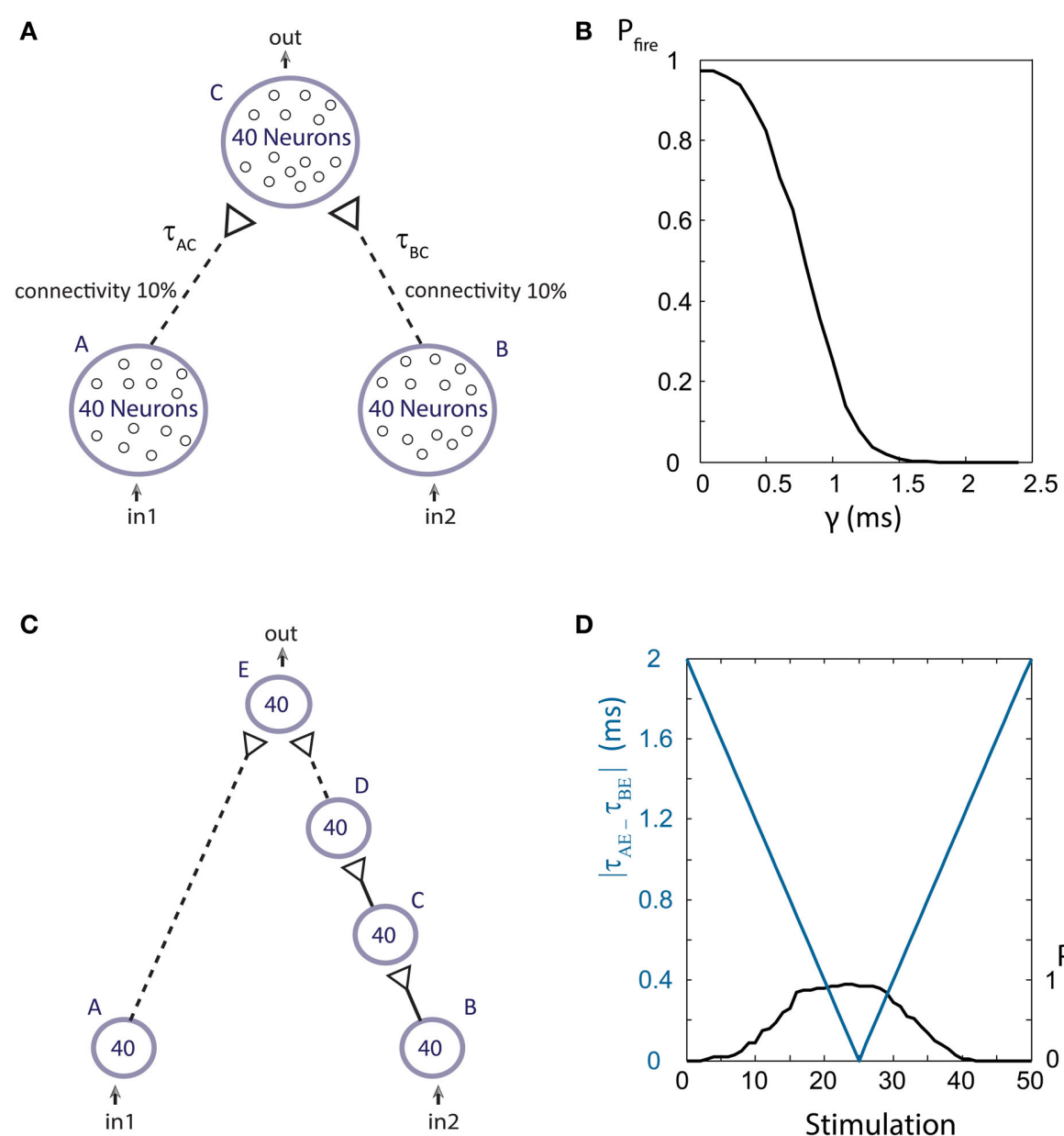

FIGURE 13 | (Color online) Population dynamics. (A) Schematic of an AND-gate in population dynamics form. Population $C$ receives week stimulations (represented by dashed arrows) from 0.1 of the neurons of each of the populations $A$ and B. (B) For simultaneous stimulations of all neurons in populations $A$ and $B$, the firing probability of the output population, $C$, is presented as a function of the time-lag between $\tau_{\mathrm{AC}}$ and $\tau_{\mathrm{BC}}, \gamma$. In the range where $\gamma$ is less than $1 \mathrm{~ms}$ an increased firing probability of population $\mathrm{C}$ is detected and the functionality of an AND-gate is maintained. (C) A dynamic

increase the stretching linearly with the number of their relays and in addition, the neuronal response latencies increase significantly faster (by one order of magnitude) in the initial spiking activity (first state,Figure 1A; Vardi et al., 2013b). Both of these biological ingredients are expected to significantly shorten mode's durations.

\section{POPULATION DYNAMICS}

The reliability of the DLGs is in question, since a finite probability of a neuronal response failure is expected. A mechanism to enhance signal-to-noise ratio can be achieved using population dynamics (Abeles, 1991; Buzsáki, 2010; Kanter et al., 2011; Kopelowitz et al., 2012). In a set of simulation studies composed of Hodgkin-Huxley neurons (Hodgkin and Huxley, 1952) at the population dynamics level we demonstrated that the time-dependent features of the new logic-gates remain valid. It is also expected that their functionality will become less sensitive to background fluctuations as the population representing each neuron increases (Vardi et al., 2012b). This feature is especially crucial to the realization of shorter synaptic delays, where the activity spontaneously terminates as a result of synaptic fatigue (Kawasaki et al., 2000; Ji et al., 2010) or neuronal refractory periods.

To examine the firing probability of a population stimulated by a sum of weak stimulations, we use the setup shown in Figure 13A. Populations A, B, and C are comprised of 40 Hodgkin-Huxley neurons with parameters similar to those in Kanter et al. (2011), where the synaptic reversal potential was set to be $\mathrm{E}_{\mathrm{syn}}=0$ and the maximal synaptic conductance for weak synaptic strengths, $g_{\max }$, was set to $0.0662 \mathrm{mS} / \mathrm{cm}^{2}$. Each neuron in population $\mathrm{C}$ was connected with probability of 0.1 to neurons in populations $\mathrm{A}$ and $\mathrm{B}$, resulting in an average of 8 input stimulations for each neuron in population $\mathrm{C}$. These diluted 
population-population stimulations, represented by the dashed arrows, are weak stimulations. Thus, to generate a spike in an output neuron, almost all stimulations from both populations A and $B$ at a sufficiently small time-lag are required, as discussed in Vardi et al. (2013b). The delays between neurons are taken from a Gaussian distribution with a standard deviation of $0.15 \mathrm{~ms}$ centered at $\tau_{\mathrm{AC}}$ and $\tau_{\mathrm{BC}}=\tau_{\mathrm{AC}}+\gamma$, where $\gamma$ is the time lag between stimulations from populations $\mathrm{A}$ and $\mathrm{B}$. The spiking probability of population $\mathrm{C}$ is measured as a function of the time-lag $\mathrm{g}$ (Figure 13B), indicating that for $\gamma<1 \mathrm{~ms}$ more than half of the neurons comprising population $\mathrm{C}$ fire for a common drive to the input populations, $\mathrm{A}$ and $\mathrm{B}$.

To demonstrate the dynamic AND-gate we construct a similar setup, containing a synfire chain from population B to population $\mathrm{E}$ (Figure 13C), where $\mathrm{g}_{\max }=1.6 \mathrm{mS} / \mathrm{cm}^{2}$ for strong synaptic strengths. The initial time delays between population are taken from a Gaussian distribution with a standard deviation of $0.15 \mathrm{~ms}$. The neuronal response latency increase per evoked spike is taken to be $\Delta=0.04 \mathrm{~ms}$ per spike (to reduce computation complexity). Simultaneous stimulations are given to all neurons in the input populations A and B. Initially, the difference $\left|\tau_{\mathrm{AE}}-\tau_{\mathrm{BE}}\right|$ is $\sim 2 \mathrm{~ms}$, therefore no output spikes are expected. As the delays between neuronal populations increase (as a result of the increase in the neuronal response latency of the population neurons) $\left|\tau_{\mathrm{AE}}-\tau_{\mathrm{BE}}\right|$ decreases, resulting in a population DLG, NULL-AND-NULL transitions (Figure 13D).

\section{CONCLUSION}

We proposed a new computational paradigm in which the brain consists of dynamic logic gates (DLGs) which are governed by time-dependent logic modes. The relevance of our work to the brain's functionalities has to be evaluated using many aspects including: (a) Do DLGs exist in the dynamics of a network of interconnected neurons? (b) Is the concept of DLGs robust to population dynamics and specifically to recurrent networks? (c) Is DLGs a mechanism which the brain could plausibly use to any extent and especially when it is critically rely on precise relative timing of neural activities? (d) Can one find a realistic learning mechanism, e.g., Hebb's rules, to implement DLGs?

The brain is composed of large neural networks, where neurons are interconnected via excitatory and inhibitory synapses as well as sub-threshold and above-threshold synapses. In the events of weak synapses, spatial and temporal summations of excitations are required to generate an evoked spike. Hence, the examined gate architectures have to be locally embedded in such large interconnected networks. The existence of weak synapses with high probability indicates that complex DLGs, where several input chains exist, are also expected to be a common building block of such networks. We verified that the phenomenon of DLGs is robust to population dynamics and hence it is expected to be less sensitive to unexpected fluctuations in the response timings of a single neuron. However, there are many unavoidable effects of brain activity which are not assumed to carry any significant information, e.g., synaptic noise. Is the DLGs one of these unavoidable effects? The answer is not yet clear, however, we showed that the increase in the neuronal response latency to ongoing stimulations cannot be ignored, as it may double its value and therefore affect the time dependent connectivity of a recurrent network. As for the implication of such DLGs to cognitive activities, we demonstrated some preliminary tasks such as edge detections, which obviously can be generalized to more complex tasks. Nevertheless, our work is a call for advanced in-vivo experiments and theoretical studies, which can pinpoint the existence and the importance of the suggested DLGs in various functionalities of the brain. Moreover, the proposed mechanism of DLGs opens a manifold of theoretical questions regarding advanced paradigm for the brain activity including the search for efficient local learning rules for the DLGs.

It is evident that the variety of possible DLGs is much larger than the abovementioned examples. For recurrent networks, the complexity is expected to be enhanced in comparison to feedforward networks. As opposed to feedforward networks with given simultaneous external stimulations, in recurrent networks the timings of the input stimulations are a function of the large scale activity of the entire network. One of the open theoretical questions is the number of realizable logic operations among $\mathrm{P}^{\mathrm{N}}$, where each one of the $\mathrm{N}$ gates has $\mathrm{P}$ operating modes.

On mathematical grounds, the key question is whether recurrent networks consisting of DLGs might go beyond the computation paradigm of the universal Turing machine (Turing, 1938; Maini et al., 2006; Dayan, 2009; Hodges, 2012). This challenge requires a careful mathematical definition and in particular, a definition of whether the stretching of the neuronal response latency has to be taken as continuous or discrete in comparison to the delays. Such networks represent a class of heterogeneous timedelayed networks composed of excitable units, where the delays are a function of the activity of the network itself. Practically, the question is whether a circuit composed of such new elements can be analyzed using the traditional systematic methods and tools developed for Boolean circuits. In the event that the presented dynamics is within traditional computational complexity, i.e., can be implemented using conventional computers, an interesting question is its advantages with respect to the implementation of the brain's functionalities.

\section{ACKNOWLEDGMENTS}

We thank Moshe Abeles and Eytan Domany for fruitful discussions and comments on the manuscript, as well as the computational assistance by Mathias Mahn, Igor Reidler, Yair Sahar, Alexander Kalmanovitch and Haya Brama. The authors thank Hana Arnon for invaluable technical assistance. This research was supported by the Ministry of Science and Technology, Israel.

\section{SUPPLEMENTARY MATERIAL}

The Supplementary Material for this article can be found online at: http://www.frontiersin.org/journal/10.3389/fncom. 2014.00052/abstract

\section{REFERENCES}

Abeles, M. (1991). Corticonics: Neural Circuits of the Cerebral Cortex. Cambridge University Press. doi: 10.1017/CBO9780511574566

Abeles, M., Hayon, G., and Lehmann, D. (2004). Modeling compositionality by dynamic binding of synfire chains. J. Comput. Neurosci. 17, 179-201. doi: 10.1023/B:JCNS.0000037682.18051.5f 
Aston-Jones, G., Segal, M., and Bloom, F. E. (1980). Brain aminergic axons exhibit marked variability in conduction velocity. Brain Res. 195, 215-222. doi: 10.1016/0006-8993(80)90880-X

Bakkum, D. J., Chao, Z. C., and Potter, S. M. (2008). Long-term activity-dependent plasticity of action potential propagation delay and amplitude in cortical networks. PLoS ONE 3:e2088. doi: 10.1371/journal.pone.0002088

Ballo, A. W., and Bucher, D. (2009). Complex intrinsic membrane properties and dopamine shape spiking activity in a motor axon. J. Neuroscience 29, 5062-5074. doi: 10.1523/JNEUROSCI.0716-09.2009

Buzsáki, G. (2010). Neural syntax: cell assemblies, synapsembles, and readers. Neuron 68, 362-385. doi: 10.1016/j.neuron.2010.09.023

Chavesa, M., Albertb, R., and Sontaga, E. D. (2005). Robustness and fragility of Boolean models for genetic regulatory networks. J. Theo. Bio. 235, 431-449. doi: 10.1016/j.jtbi.2005.01.023

Dayan, P. (2009). A neurocomputational jeremiad. Nat. Neuroscience 12, 1207-1207. doi: 10.1038/nn1009-1207

De Col, R., Messlinger, K., and Carr, R. W. (2008). Conduction velocity is regulated by sodium channel inactivation in unmyelinated axons innervating the rat cranial meninges. J. physiology 586, 1089-1103. doi: 10.1113/jphysiol.2007. 145383

Eccles, J. C., Llinas, R., and Sasaki, K. (1966). Excitatory synaptic action of climbing fibres on purkinje cells of cerebellum. J. Physiology 182, 268-296.

Fuhrmann, G., Markram, H., and Tsodyks, M. (2002). Spike frequency adaptation and neocortical rhythms. J. Neurophysiol. 88, 761-770. doi: 10.1007/s00422008-0244-y

Gal, A., Eytan, D., Wallach, A., Sandler, M., Schiller, J., and Marom, S. (2010). Dynamics of excitability over extended timescales in cultured cortical neurons. J. Neuroscience 30, 16332-16342. doi: 10.1523/JNEUROSCI.4859-10.2010

Gerstner, W., Sprekeler, H., and Deco, G. (2012). Theory and Simulation in Neuroscience. Science 338, 60-65. doi: 10.1126/science.1227356

Gilja, V., Nuyujukian, P., Chestek, C. A., Cunningham, J. P., Byron, M. Y., Fan, J. M., et al. (2012). A high-performance neural prosthesis enabled by control algorithm design. Nat. Neurosci. 15, 1752-1757. doi: 10.1038/nn.3265

Grossman, Y., Parnas, I., and Spira, M. E. (1979). Mechanisms involved in differential conduction of potentials at high frequency in a branching axon. J. Physiol. 295, 307-322.

Hodges, A. (2012). Beyond turing's machines. Science 336, 163-164. doi: $10.1126 /$ science. 1218417

Hodgkin, A. L., and Huxley, A. F. (1952). A quantitative description of membrane current and its application to conduction and excitation in nerve. J. Physiol. 117, 500-544.

Hopfield, J. J. (1982). Neural networks and physical systems with emergent collective computational abilities. Proc. Natl. Acad. Sci. U.S.A. 79, 2554-2558. doi: 10.1073/pnas.79.8.2554

Hunt, L. T., Kolling, N., Soltani, A., Woolrich, M. W., Rushworth, M. F. S., and Behrens, T. E. J. (2012). Mechanisms underlying cortical activity during valueguided choice. Nat. Neurosci. 15, 470-476. doi: 10.1038/nn.3017

Ikegaya, Y., Aaron, G., Cossart, R., Aronov, D., Lampl, I., Ferster, D., et al. (2004). Synfire chains and cortical songs: temporal modules of cortical activity. Science 304, 559-564. doi: 10.1126/science. 1093173

Izhikevich, E. M. (2006). Polychronization: computation with spikes. Neural Comput. 18, 245-282. doi: 10.1162/089976606775093882

Izhikevich, E. M., and Hoppensteadt, F. C. (2009). Polychronous wavefront computations. Int. J. Bif. Chaos 19, 1733-1739. doi: 10.1142/S0218127409023809

Ji, Y. Y., Lu, Y., Yang, F., Shen, W. H., Tang, T. T. T., Feng, L. Y., et al. (2010). Acute and gradual increases in BDNF concentration elicit distinct signaling and functions in neurons. Nat. Neurosci. 13, 302-309. doi: 10.1038/nn.2505

Kanter, I., Kopelowitz, E., Vardi, R., Zigzag, M., Kinzel, W., Abeles, M., et al. (2011). Nonlocal mechanism for cluster synchronization in neural circuits. EPL 93:66001. doi: 10.1209/0295-5075/93/66001

Kawasaki, F., Hazen, M., and Ordway, R. W. (2000). Fast synaptic fatigue in shibire mutants reveals a rapid requirement for dynamin in synaptic vesicle membrane trafficking. Nat. Neurosci. 3, 859-860. doi: 10.1038/78753

Kopelowitz, E., Abeles, M., Cohen, D., and Kanter, I. (2012). Sensitivity of global network dynamics to local parameters versus motif structure in a cortexlike neuronal model. Phys. Rev. E 85:051902. doi: 10.1103/PhysRevE.85. 051902

Krogh, A. (2008). What are artificial neural networks? Nat. Biotechnol. 26, 195-197. doi: $10.1038 /$ nbt1386
Litwin-Kumar, A., and Doiron, B. (2012). Slow dynamics and high variability in balanced cortical networks with clustered connections. Nat. Neurosci. 15, 1498-1505. doi: 10.1038/nn.3220

Long, M. A., Jin, D. Z., and Fee, M. S. (2010). Support for a synaptic chain model of neuronal sequence generation. Nature 468, 394-399. doi: 10.1038/nature09514

Maini, P. K., Baker, R. E., and Chuong, C. (2006). The turing model comes of molecular age. Science 314, 1397. doi: 10.1126/science.1136396

Markram, H., and Tsodyks, M. (1996). Redistribution of synaptic efficacy between neocortical pyramidal neurons. Nature 382, 807-810. doi: 10.1038/382807a0

Marom, S., and Shahaf, G. (2002). Development, learning and memory in large random networks of cortical neurons: lessons beyond anatomy. Q. Rev. Biophys. 35, 63-87. doi: 10.1017/S0033583501003742

Mcculloch, W. S., and Pitts, W. (1943). A logical calculus of the ideas immanent in nervous activity. Bull. Math. Biophys. 5, 115-133. doi: 10.1007/BF02478259

Morin, F. O., Takamura, Y., and Tamiya, E. (2005). Investigating neuronal activity with planar microelectrode arrays: achievements and new perspectives. J. Biosci. Bioeng. 100, 131-143. doi: 10.1263/jbb.100.131

Nahin, P. J. (2012). The Logician and The Engineer: How George Boole and Claude Shannon Created the Information Age. Princeton, NJ: Princeton Univ. Press.

Pastalkova, E., Itskov, V., Amarasingham, A., and Buzsáki, G. (2008). Internally generated cell assembly sequences in the rat hippocampus. Science 321, 1322-1327. doi: $10.1126 /$ science. 1159775

Qian, L., Winfree, E., and Bruck, J. (2011). Neural network computation with DNA strand displacement cascades. Nature 475, 368-372. doi: 10.1038/nature 10262

Rosenblatt, F. (1958). The perceptron: a probabilistic model for information storage and organization in the brain. Psychol. Rev. 65, 386. doi: 10.1037/h0042519

Scroggs, R. S. (2008). Evidence of a physiological role for use-dependent inactivation of navl.8 sodium channels. J. Physiol. 586, 923. doi: 10.1113/jphysiol.2008.150821

Shannon, C. (1938). A symbolic analysis of relay and switching circuits. Trans. AIEE 57, 713-723.

Soudry, D., and Meir, R. (2012). Conductance-based neuron models and the slow dynamics of excitability. Front. Neurosci. 6:4. doi: 10.3389/fncom.2012.00004

Spira, M. E., Yarom, Y., and Parnas, I. (1976). Modulation of spike frequency by regions of special axonal geometry and by synaptic inputs. J. Neurophysiol. 39, 882-899.

Stoianov, I., and Zorzi, M. (2012). Emergence of a "visual number sense" in hierarchical generative models. Nat. Neurosci. 15, 194-196. doi: 10.1038/nn.2996

Sutton, R. S., and Barto, A. G. (1998). Reinforcement Learning: An Introduction. Cambridge: Cambridge Univ Press.

Tal, D., Jacobson, E., Lyakhov, V., and Marom, S. (2001). Frequency tuning of inputoutput relation in a rat cortical neuron in-vitro. Neurosci. Lett. 300, 21-24. doi: 10.1016/S0304-3940(01)01534-8

Thomson, A. M., and West, D. C. (1993). Fluctuations in pyramid excitatory postsynaptic potentials modified by presynaptic firing pattern and postsynaptic membrane-potential using paired intracellular-recordings in rat neocortex. Neuroscience 54, 329-346. doi: 10.1016/0306-4522(93)90256-F

Turing, A. M. (1938). "On computable numbers, with an application to the Entscheidungsproblem. A Correction," in Proceedings of the London Mathematical Society (London), Ser. 2, 544. doi: 10.1112/plms/s2-43.6.544

Van Pelt, J., Wolters, P. S., Corner, M. A., Rutten, W. L. C., and Ramakers, G. J. A. (2004). Long-term characterization of firing dynamics of spontaneous bursts in cultured neural networks. IEEE Trans. Biomed. Eng. 51, 2051-2062. doi: 10.1109/TBME.2004.827936

Vardi, R., Goldental, A., Guberman, S., Kalmanovich, A., Marmari, H., and Kanter, I. (2013a). Sudden synchrony leaps accompanied by frequency multiplications in neuronal activity. Front. Neural Circuits 7:176. doi: 10.3389/fncir.2013.00176

Vardi, R., Guberman, S., Goldental, A., and Kanter, I. (2013b). An experimental evidence-based computational paradigm for new logic-gates in neuronal activity. EPL 103:66001. doi: 10.1209/0295-5075/103/66001

Vardi, R., Timor, R., Marom, S., Abeles, M., and Kanter, I. (2012a). Synchronization with mismatched synaptic delays: a unique role of elastic neuronal latency. EPL 100:48003. doi: 10.1209/0295-5075/100/48003

Vardi, R., Timor, R., Marom, S., Abeles, M., and Kanter, I. (2013c). Synchronization by elastic neuronal latencies. Phys. Rev. E 87:012724. doi: 10.1103/PhysRevE.87.012724

Vardi, R., Wallach, A., Kopelowitz, E., Abeles, M., Marom, S., and Kanter, I. (2012b). Synthetic reverberating activity patterns embedded in networks of cortical neurons. EPL 97:66002. doi: 10.1209/0295-5075/97/66002 
Vogels, T. P., and Abbott, L. F. (2005). Signal propagation and logic gating in networks of integrate-and-fire neurons. J. Neurosci. 25, 10786-10795. doi: 10.1523/JNEUROSCI.3508-05.2005

Von Neumann, J. (1956). Probabilistic logics and the synthesis of reliable organisms from unreliable components. Automata Stud. 34, 43-98.

Wagenaar, D., Pine, J., and Potter, S. (2006). An extremely rich repertoire of bursting patterns during the development of cortical cultures. BMC Neurosci. 7:11. doi: 10.1186/1471-2202-7-11

Conflict of Interest Statement: The authors declare that the research was conducted in the absence of any commercial or financial relationships that could be construed as a potential conflict of interest.
Received: 03 February 2014; paper pending published: 31 March 2014; accepted: 07 April 2014; published online: 29 April 2014.

Citation: Goldental A, Guberman S, Vardi R and Kanter I (2014) A computational paradigm for dynamic logic-gates in neuronal activity. Front. Comput. Neurosci. 8:52. doi: $10.3389 /$ fncom.2014.00052

This article was submitted to the journal Frontiers in Computational Neuroscience. Copyright (C) 2014 Goldental, Guberman, Vardi and Kanter. This is an open-access article distributed under the terms of the Creative Commons Attribution License (CC BY). The use, distribution or reproduction in other forums is permitted, provided the original author(s) or licensor are credited and that the original publication in this journal is cited, in accordance with accepted academic practice. No use, distribution or reproduction is permitted which does not comply with these terms. 